\title{
Spatial Tuning Shifts Increase the Discriminability and Fidelity of Population Codes in Visual Cortex
}

\author{
다 A. Vo, ${ }^{1}$ Thomas C. Sprague, ${ }^{1,2}$ and - John T. Serences ${ }^{1,3,4}$ \\ ${ }^{1}$ Neurosciences Graduate Program, University of California, San Diego, La Jolla, California 92093, ${ }^{2}$ Department of Psychology and Center for Neural Science, \\ New York University, New York, New York 10003, Department of Psychology, University of California, San Diego, La Jolla, California 92093, \\ and ${ }^{4}$ Kavli Institute for Brain and Mind, University of California, San Diego, La Jolla, California 92093
}

Selective visual attention enables organisms to enhance the representation of behaviorally relevant stimuli by altering the encoding properties of single receptive fields (RFs). Yet we know little about how the attentional modulations of single RFs contribute to the encoding of an entire visual scene. Addressing this issue requires (1) measuring a group of RFs that tile a continuous portion of visual space, (2) constructing a populationlevel measurement of spatial representations based on these RFs, and (3) linking how different types of RF attentional modulations change the population-level representation. To accomplish these aims, we used fMRI to characterize the responses of thousands of voxels in retinotopically organized human cortex. First, we found that the response modulations of voxel RFs (vRFs) depend on the spatial relationship between the RF center and the visual location of the attended target. Second, we used two analyses to assess the spatial encoding quality of a population of voxels. We found that attention increased fine spatial discriminability and representational fidelity near the attended target. Third, we linked these findings by manipulating the observed vRF attentional modulations and recomputing our measures of the fidelity of population codes. Surprisingly, we discovered that attentional enhancements of population-level representations largely depend on position shifts of vRFs, rather than changes in size or gain. Our data suggest that position shifts of single RFs are a principal mechanism by which attention enhances populationlevel representations in visual cortex.

Key words: attention; encoding models; labeled lines; population codes; receptive fields; vision

\section{Significance Statement}

Although changes in the gain and size of RFs have dominated our view of how attention modulates visual information codes, such hypotheses have largely relied on the extrapolation of single-cell responses to population responses. Here we use fMRI to relate changes in single voxel receptive fields (vRFs) to changes in population-level representations. We find that vRF position shifts contribute more to population-level enhancements of visual information than changes in vRF size or gain. This finding suggests that position shifts are a principal mechanism by which spatial attention enhances population codes for relevant visual information. This poses challenges for labeled line theories of information processing, suggesting that downstream regions likely rely on distributed inputs rather than single neuron-to-neuron mappings.

\section{Introduction}

Spatial receptive fields (RFs) are a core component of visual information processing throughout the visual system. They are modified by selective visual attention to improve the fidelity of

\footnotetext{
Received Nov. 9, 2016; revised Feb. 13, 2017; accepted Feb. 16, 2017.

Author contributions: V.A.V., T.C.S., and J.T.S. designed research; V.A.V. and T.C.S. performed research; V.A.V., T.C.S., and J.T.S. contributed unpublished reagents/analytic tools; V.A.V. and T.C.S. analyzed data; V.A.V. wrote the paper.

This work was supported by National Science Foundation Graduate Research Fellowships to V.A.V. and T.C.S. National Eye Institute Grant R01-EY025872, and James S. McDonnell Foundation Scholar Award to J.T.S. We thank the lab, Rosanne Rademaker, and Edward Vul for comments on analyses and on the manuscript.

The authors declare no competing financial interests.

Correspondence should be addressed to either Vy A. Vo or John T. Serences, Neurosciences Graduate Program, University of California, San Diego, La Jolla, CA 92093-0634. E-mail: vyaivo@ucsd.edu or jserences@ucsd.edu.

DOI:10.1523/JNEUROSCI.3484-16.2017

Copyright $\odot 2017$ the authors $\quad 0270-6474 / 17 / 373386-16 \$ 15.00 / 0$
}

sensory representations, likely enabling more precise, accurate perception (Desimone and Duncan, 1995; Anton-Erxleben and Carrasco, 2013). Prior studies in nonhuman primates have found that covert spatial attention changes the position, size, and amplitude of responses in single-cell RFs in early cortical areas, such as V1, V4, and MT (Moran and Desimone, 1985; Connor et al., 1996, 1997; Womelsdorf et al., 2006, 2008; Roberts et al., 2007; David et al., 2008). Recent neuroimaging studies have also shown that single-voxel RFs (vRFs) undergo similar response changes with attention, shifting toward the attended target or changing in size (Klein et al., 2014; de Haas et al., 2014; Kay et al., 2015; Sheremata and Silver, 2015). Most accounts suggest that these RF modulations improve the spatial representations of the attended target, either by boosting the signal-to-noise ratio by increasing response amplitude, or by increasing the spatial resolution by decreas- 
ing RF size (Desimone and Duncan, 1995; Anton-Erxleben and Carrasco, 2013; Cohen and Maunsell, 2014). These mechanisms are akin to turning up the volume (gain increase) or to using smaller pixels to encode a digital image (size decrease).

Despite these documented modulations, it is not yet clear how different types of RF modulations are combined to facilitate robust population codes. Recent studies have only begun to explore how interactions between neurons may affect the coding properties of the population (Anton-Erxleben and Carrasco, 2013; Cohen and Maunsell, 2014). Yet analyzing these data at a population level is crucial for understanding how spatial attention changes the overall representation of an attended area. Prior fMRI studies that measured many voxel RFs (vRFs) across space were often unable to report the full pattern of response modulations with respect to the attended target because subjects attended to the mapping stimulus, rather than to a fixed point in space (Sprague and Serences, 2013; Kay et al., 2015; Sheremata and Silver, 2015). Studies that fixed the locus of attention have reported mixed results on vRF modulations (Klein et al., 2014; de Haas et al., 2014). The first aim of this study was thus to evaluate how properties of vRFs in retinotopic areas change with attention, especially near the peripheral attention target.

The second aim of the study was to evaluate how different types of RF modulations contribute to population-level enhancements of an attended region of space. Single RFs in early visual areas are fundamentally local encoding models that are relatively uninformative about regions outside their immediate borders. To study their relationship to a population-level representation of space, other metrics are needed to integrate information across all local encoding units (e.g., vRFs) to evaluate how attentional modulations impact the quality of population codes. Here, we used two different population-level metrics of spatial encoding fidelity to investigate these questions, and to determine how changes in vRF amplitude, size, or position affect the populationlevel representations. First, we used a measure related to Fisher information to evaluate the spatial discriminability of population codes. Second, we used a spatial encoding model that incorporates information across voxels to form representations of stimuli in the mapped visual field (Brouwer and Heeger, 2009; Sprague and Serences, 2013; Sprague et al., 2015).

We found that vRF position shifts increase both the spatial discriminability around the attended region as well as the fidelity of stimulus reconstructions near the attended target. Surprisingly, shifts in vRF position accounted for more of the population-level enhancements with attention than changes in vRF size or gain. This finding is unexpected in the context of "labeled-line" models of information processing, which posit that visual representations rely on RFs that transmit consistent "labels" for visual features, such as spatial position. Our findings suggest that apparent shifts in the labels of RFs play an important role in the attentional enhancement of visual information.

\section{Materials and Methods}

\section{Task design and participants}

We collected data from 9 human participants ( 4 female), 6 of whom had previously completed a set of retinotopic mapping scans in the laboratory (Participants AA, AB, AC, AI, and AL in Sprague and Serences, 2013; Participants AA, AC, and AI in Sprague et al., 2014; all participants in Ester et al., 2015). All participants provided written informed consent and were compensated for their time $(\$ 20 / \mathrm{h})$ as approved by the local University of California, San Diego Institutional Review Board. Participants practiced both the attention task and the localizer task before entering the scanner. A minimum of $4 \mathrm{~h}$ of scanning was required to complete the entire analysis, so one participant was excluded due to insufficient data (they only completed $2 \mathrm{~h}$ ). Another participant was excluded for inconsistent behavioral performance, with average task accuracy at chance $(48.6 \%)$. This yielded a total of 7 participants who completed the entire experiment ( $32 \mathrm{~h}$ scan sessions per participant).

Participants centrally fixated a gray rectangular screen $(120 \times 90 \mathrm{~cm})$ viewed via a head-coil mounted mirror ( $\sim 3.85 \mathrm{~m}$ viewing distance). They attended one of three fixed locations on the screen: the fixation point or a target to the lower left or lower right of fixation. During each $2000 \mathrm{~ms}$ trial, subjects reported a change in the attention target. When subjects attended fixation, they reported whether a brief contrast change (100-400 ms, starting 300-1000 ms into the trial) was dimmer or brighter than the baseline contrast. The peripheral attention targets were two pentagons $\left(0.17^{\circ}\right.$ radius; $50 \%$ contrast $)$ centered $2.1^{\circ}$ to the left and right of fixation (Fig. 1a). When subjects attended a peripheral target, they reported whether it rotated clockwise or counterclockwise (rotation duration 100-300 ms, starting 300-1600 ms into the trial). Intertrial intervals randomly varied between 1000 and $3000 \mathrm{~ms}$ in $500 \mathrm{~ms}$ increments (mean intertrial interval: $2000 \mathrm{~ms}$ ). The magnitude of the contrast change or the rotation was adjusted on each run to keep task performance for each participant near $75 \%$ (mean $=75.90 \%$, bootstrapped $95 \%$ CI $[72.46 \%, 79.20 \%]$ ), with no significant difference between conditions as evaluated with a one-way repeated-measures ANOVA randomization test $\left(F_{(1,11)}=0.220\right.$, randomized $\left.p=0.800\right)$. For 4 participants, we collected 6 runs on the attend periphery tasks without a change in the luminance of the fixation stimulus. Performance on the attend periphery tasks was stable across runs with and without the luminance change (repeated-measures ANOVA with run type $\times$ random participants factor; $p=0.439$, null $F$ distribution using randomized labels for 10,000 iterations). Therefore, these data were collapsed across scan sessions with and without changes in fixation luminance.

On 51 of the 61 trials in each run, a full-contrast $6 \mathrm{~Hz}$ flickering checkerboard $\left(0.68^{\circ}\right.$ radius; 1.67 cycles/deg) appeared for $2000 \mathrm{~ms}$ at 1 of 51 different locations across the screen to map the spatial sensitivity of visually responsive voxels. These mapping stimuli covered a region of the screen approximately subtending $9^{\circ}$ horizontal and $6^{\circ}$ vertical when their position was jittered. When one of these checkerboards overlapped with any of the static attention targets, they were partially masked with a small circular aperture the same color as the screen background $\left(0.16^{\circ} / 0.25^{\circ}\right.$ radius aperture for fixation/ pentagon, respectively) that allowed the stimulus to remain visible. Participants were instructed to ignore the task-irrelevant flickering checkerboards throughout the experiment. During the 10 null trials on each scan, the participant continued to perform the attention task but no checkerboard was presented. Null trials and mapping stimulus trials were presented in a pseudorandom interleaved order.

The location of the checkerboard mapping stimulus on each trial was determined by generating an evenly spaced triangular grid $\left(0.84^{\circ}\right.$ between grid points) and centering the checkerboard on one of these grid points. The location of the checkerboard mapping stimulus was then jittered a random amount from these grid points $\left( \pm 0.42^{\circ} / 0.37^{\circ}\right.$ horizon$\mathrm{tal} /$ vertical). When subjects attended the peripheral target, half of the runs were presented at the discrete grid positions so that we could achieve more stable stimulus reconstructions (see Stimulus reconstructions using an inverted spatial encoding model).

\section{MRI}

We obtained all structural and functional MR images using a GE 3T MR750 scanner at the University of California, San Diego. We collected all functional images $(19.2 \mathrm{~cm} \times 19.2 \mathrm{~cm}$ FOV, $64 \times 64$ acquisition matrix, 35 interleaved slices, $3 \mathrm{~mm}^{3}$ voxels with $0 \mathrm{~mm}$ slice gap, 128 volumes per scan run) using a gradient echo planar pulse sequence (2000 $\mathrm{ms} \mathrm{TR}, 30 \mathrm{~ms} \mathrm{TE}, 90^{\circ} \mathrm{flip}$ angle) and a 32-channel head coil (Nova Medical). Five dummy scans preceded each functional run. A highresolution structural image was acquired at the end of each session using a FSPGR T1-weighted pulse sequence $(25.6 \mathrm{~cm} \times 25.6 \mathrm{~cm}$ FOV, $256 \times$ 192 acquisition matrix, 8.136/3.172 ms TR/TE, 192 slices, $9^{\circ}$ flip angle, 1 $\mathrm{mm}^{3}$ voxels). All functional scans were coregistered to the anatomical images acquired during the same session, and this anatomical was in turn coregistered to the anatomical acquired during the retinotopy scan.

EPI images were unwarped with a custom script from UCSD's Center for Functional Magnetic Resonance Imaging using FSL and AFNI. All 
a

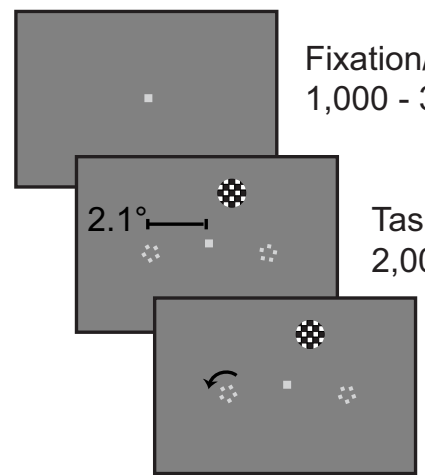

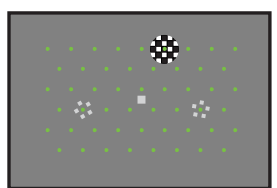

Mapping probe locations

$0.84^{\circ}$ apart

jittered $+/-0.42^{\circ}$

\section{b Estimate voxel RF (VRF) parameters}

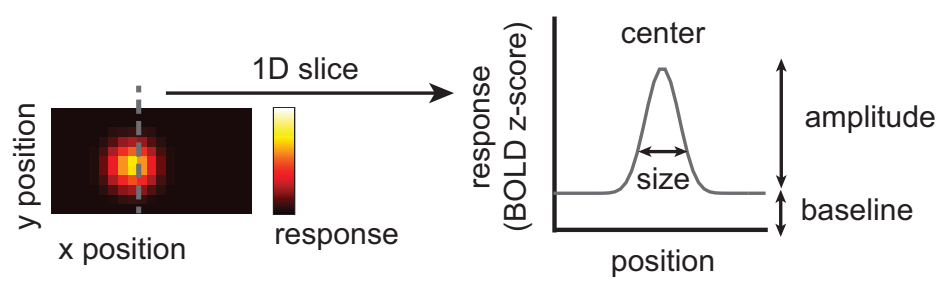

C Estimate how attentional modulations in vRFs affect population measures

\section{Local spatial discriminability}

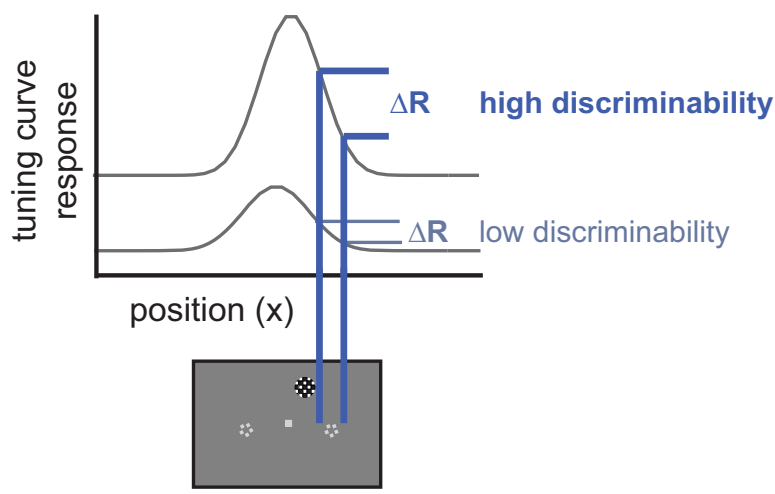

Inverted encoding model (IEM)

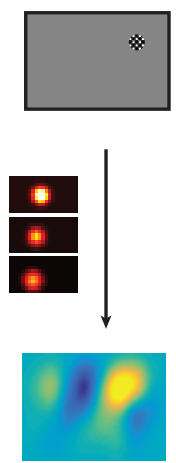

Example trial

Combine vRFs into population-level encoding model

Stimulus reconstruction

Figure 1. Covert spatial attention task and fMRI-based analyses used to link single voxels to population-level measurements. $\boldsymbol{a}$, Subjects fixated centrally and attended to brief rotations in the pentagon stimulus on the left or right while a flickering checkerboard probe stimulus appeared at 1 of 51 grid locations across the visual field. On control runs, subjects attended to a contrast change at fixation. fMRI data measured during this attention task are used to create visualizable estimates of vRFs and stimulus reconstructions. $\boldsymbol{b}$, A receptive field model is fit to the responses of each voxel and can be described by its $x$ and $y$ position (center), response baseline, response amplitude, and size (FWHM). c, Given a population of voxels in a retinotopic region, such as V1, we examine two different measures of spatial information in the population. The first, a spatial discriminability metric, scales with the slope of the tuning curve at a given location in space (see Materials and Methods). The second relies on a multivariate IEM for space. By reconstructing images of the mapping stimulus on each test trial, we can measure how population-level spatial information changes with attention. We then can model how changes in individual vRFs affect both of these population measures.

subsequent preprocessing was performed in BrainVoyager 2.6.1, including slice-time correction, six-parameter affine motion correction, and temporal high-pass filtering to remove slow signal drifts over the course of each run. Data were then transformed into Talairach space and resampled to have a $3 \times 3 \times 3 \mathrm{~mm}$ voxel size. Finally, the BOLD signal in each voxel was transformed into $z$ scores on a scan-by-scan basis. All subsequent analyses were performed in MATLAB using custom scripts (available online on Open Science Framework: osf.io/s9vqv).

\section{Independent localizer task}

We constrained our analyses to visually responsive voxels in occipital and parietal cortex using a separate localizer task (3-5 runs per participant). On 14 trials, participants fixated centrally and viewed a full-field flickering checkerboard $\left(10 \mathrm{~Hz}, 11.0 / 8.3^{\circ}\right.$ width/height $)$ for $8000 \mathrm{~ms}$. Participants detected whether a small area (2D Gaussian, $\sigma=0.2^{\circ}$ ) within the checkerboard dimmed in contrast. Contrast dimming occurred between 500 and $4000 \mathrm{~ms}$ after the start of the trial and lasted between 2000 and
$3000 \mathrm{~ms}$ (all uniformly sampled in $500 \mathrm{~ms}$ steps). This contrast change occurred infrequently (randomly on 5 of 14 trials) at a random location within the checkerboard. The average contrast change was varied between runs to maintain consistent performance at $\sim 75 \%$ accuracy (mean performance $78.0 \%$ ). On 8 trials, participants simply fixated throughout the trial without a checkerboard being presented. We then used a standard GLM with a canonical two-gamma HRF (peak at $5 \mathrm{~s}$, undershoot peak at $15 \mathrm{~s}$, response undershoot ratio 6 , response dispersion 1, undershoot dispersion 1) to estimate the response to the checkerboard stimulus in each voxel. For all subsequent analyses, only voxels in the retinotopically defined areas V1, V2, V3, V4, V3A/B, and IPS0 with a significantly positive BOLD response to the localizer task (at FDR $q=$ 0.05) were included (Benjamini and Yekutieli, 2001).

\section{Estimating single-trial BOLD responses}

For all subsequent analyses, we used trial-wise BOLD $z$ scores. We estimated these by creating a boxcar model marking the duration of each 
Table 1. vRF selection statistics, pooled across participants $(N=7)$

\begin{tabular}{|c|c|c|c|c|c|c|c|}
\hline ROI & $\begin{array}{l}\text { Total no. of } \\
\text { localized voxels }\end{array}$ & $\begin{array}{l}\text { No. of voxels after } \\
\text { GLM thresholding }\end{array}$ & $\begin{array}{l}\text { No. of voxels after } \\
\text { regularizability threshold }\end{array}$ & $\begin{array}{l}\text { No. of voxels after } \\
\text { cross-validation threshold }\end{array}$ & $\begin{array}{l}\text { No. of voxels after } \\
\text { removing difference } \\
\text { score outliers }\end{array}$ & $\begin{array}{l}\text { Percentage that survive } \\
\text { all thresholds }\end{array}$ & $\begin{array}{l}\text { RMSE fit error for } \\
\text { surviving voxels }\end{array}$ \\
\hline V1 & 3723 & 3540 & 2438 & 989 & 931 & 25.01 & 0.1105 \\
\hline V2 & 4154 & 3970 & 3115 & 1405 & 1339 & 32.23 & 0.1087 \\
\hline V3 & 3698 & 3519 & 2839 & 1520 & 1435 & 38.81 & 0.0994 \\
\hline V4 & 1702 & 1492 & 1118 & 361 & 336 & 19.74 & 0.0783 \\
\hline $\mathrm{V} 3 \mathrm{~A} / \mathrm{B}$ & 1988 & 1922 & 1440 & 443 & 416 & 20.93 & 0.0893 \\
\hline IPSO & 1567 & 1492 & 800 & 114 & 110 & 7.02 & 0.0882 \\
\hline V1-V4 & 13,277 & 12,521 & 9510 & 4275 & 4041 & 30.44 & 0.1032 \\
\hline V3A/B and IPSO & 3555 & 3414 & 2240 & 557 & 526 & 14.80 & 0.0894 \\
\hline Total & 16,832 & 15,935 & 11,750 & 4832 & 4567 & 27.13 & 0.1016 \\
\hline
\end{tabular}

checkerboard mapping stimulus and convolving it with a canonical twogamma HRF (peak at $5 \mathrm{~s}$, undershoot peak at $15 \mathrm{~s}$, response undershoot ratio 6 , response dispersion 1 , undershoot dispersion 1 ). To standardize our data across runs, we $z$ scored the BOLD responses within each run and concatenated the $z$ scores across runs. We then solved a GLM to find the response to each predictor.

\section{Statistical procedures}

All reported CIs are computed by resampling the data with replacement (i.e., bootstrapping). The number of iterations for each bootstrapping procedure varied (depending on available computing power and time for that procedure) and are therefore reported with each result. For tests comparing a bootstrapped distribution against zero, $p$ values were computed by conducting two one-tailed tests against 0 (e.g., mean( param_change $<0$ ) and mean ( param_change $>0)$ ) and doubling the smaller $p$ value. All repeated tests were FDR corrected $(q=0.05)$.

\section{$v R F$ estimation, fitting, and parameter analysis}

We first estimated vRFs for each attention condition to investigate (1) how vRF parameters changed when participants attended to different locations and (2) the spatial pattern vRF changes across visual space. We note here that prior reports have referred to similar vRF models as population receptive fields to emphasize the fact that each voxel contains a population of spatially tuned neurons (Dumoulin and Wandell, 2008; Wandell and Winawer, 2015). However, because we are comparing modulations at different scales in the present study (i.e., modulations in single voxels and in patterns of responses across many voxels), we will refer to these single voxel measurements as vRFs, and will reserve the term "population" exclusively for multivariate measures involving several voxels, allowing our terminology to align with theories of population coding (Ma et al., 2006).

We estimated vRFs using a modified version of a previously described technique (Sprague and Serences, 2013). This method estimates a single voxel's spatial sensitivity by modeling its BOLD responses as a linear combination of discrete, smooth spatial filters tiled evenly across the mapped portion of the visual field. These spatial filters (or spatial channels) form our modeled basis set. We then regressed the BOLD $z$ scores $(v$ voxels $\times n$ trials) onto a design matrix with predicted channel responses for each trial ( $C$, $k$ channels $\times n$ trials) by solving Equation 1 as follows:

$$
B=W C
$$

for the matrix $W$ ( $v$ voxels $\times k$ channels).

Each of the $k$ channels in the basis set was defined as a $2 \mathrm{D}$ cosine that was fixed to reach 0 at a set distance from the filter center as follows:

$$
f(r)=\left(0.5\left(\cos \left(\frac{r \pi}{s}\right)+0.5\right)\right)^{7} \quad \text { for } r<s
$$

where $r$ is the distance from the filter center and $s$ is the size constant. Setting a zero baseline in this function ensured that we could estimate a stable baseline for each voxel by restricting the response of the channel to a known subregion of the visual display. Because the estimated vRF size depends on the size of the filters, we made the filters fairly small $\left(1.08^{\circ}\right.$ FWHM) and dense (91 filters arranged in a 13 horizontal/7 vertical grid, each spaced $0.83^{\circ}$ apart). We then discretized the filters by sampling them in a high-resolution $2 \mathrm{D}$ grid of $135 \times 101$ pixels spanning $10^{\circ} \times 5^{\circ}$. The discretized filters ( $k$ filters $\times p$ pixels) were multiplied with a mask of the checkerboard stimulus on every trial ( $p$ pixels $\times n$ trials) so that the design matrix $C$ contained predictions of the spatial channel responses on every trial of the mapping task.

To fit our estimated vRFs with a unimodal function, we used ridge regression to solve Equation 1. This is a common regularization method that sparsifies the regression solution by penalizing the regressors with many small weights (Hoerl and Kennard, 1970; Lee et al., 2013). This meant solving for an estimate of $W$ by the following:

$$
\hat{W}^{T}=\left(C C^{T}+\lambda I\right)^{-1} C B^{T}
$$

where $\lambda$ is the ridge parameter penalty term and $I$ is a $k \times k$ identity matrix. We estimated an optimal $\lambda$ for each voxel by evaluating Equation 3 over a range of $\lambda$ values $(0-750)$ for a balanced number of runs of the attention task (e.g., an equal number of runs from each attention condition). We then computed the Bayesian Information Criterion for each of these $\lambda$ values, estimating the degrees of freedom in the ridge regression as follows:

$$
d f=\operatorname{trace}\left(C\left(C^{T} C+\lambda I\right)^{-1} C^{T}\right)
$$

The $\lambda$ with the smallest Bayesian Information Criterion was selected for each voxel. Because the attention comparisons are done within voxels, the varying $\lambda$ penalty across voxels could not explain the attention effects we observed.

To select reliable voxels for analysis, we next implemented a set of conservative thresholding steps (Table 1). We first needed to select voxels with reliable visual responses, so we only kept voxels with trial $\beta$ weights that predicted at least $50 \%$ of the BOLD time courses in each scan session. Second, we only used voxels that could be successfully regularized with ridge regression. Any voxels with the maximum $\lambda$ (750) were discarded, as this indicated that the ridge regression solution had not converged. Finally, we verified that the resulting regression model could predict an independent dataset, so we performed exhaustive leave-one-run-out cross validation for each attention condition. This ensured that the $\lambda$ estimated across attention conditions produced reliable data for each condition separately. We estimated $W$ using data from all but one run (Eq. 3) and used this to predict the BOLD GLM trial estimate of the left-out run (Eq. 2), separately for each condition. We then computed the mean correlation between the predicted and real BOLD GLM trial estimates across cross-validation folds for each voxel. It is not possible to calculate a coefficient of determination on regularized data because the process of ridge regression changes the scale of the predicted data (Huth et al., 2012). We only kept voxels where this cross-validation $r>0.25$ for all 3 conditions.

To quantify each vRF, we fit the spatial RF profile of each voxel with a smooth 2D function with four parameters: center, size, baseline, and amplitude (Fig. 1b; Eq. 2). Here, we define the vRF baseline as the voxel's response that does not reliably depend on the position of the mapping stimulus (i.e., its constant offset). The vRF amplitude is defined as the spatially selective increase in a voxel's response above this baseline. To- 
gether, these two parameters index how much of the voxel's response is due to a change in mapping stimulus position. Finally, the size and location parameters estimate the spatial selectivity and the spatial position preference of the vRFs, respectively. We first downsampled the vRFs by multiplying the estimated weights $\hat{W}$ for each voxel $($ a $1 \times k$ channel vector) by a smaller version of the spatial grid that contained the basis set $\left(68 \times 51\right.$ pixel grid; $\left.10^{\circ} \times 5^{\circ}\right)$. This speeded up the process of fitting the pixelwise surface with Equation 2. This fitting process began with a coarse grid search that first found the best fit in a discrete grid of possible vRF parameters (center sampled in $1^{\circ}$ steps over the mapped portion of the visual field; size constant logarithmically sampled at 20 points between FWHM of $10^{0.01}$ degrees and $10^{1}$ degrees). At each grid point, we estimated the best fit amplitude and baseline using linear regression. The grid point fit with the smallest root mean square error (RMSE) provided the initialization seed to a continuous error function optimization algorithm (fmincon in MATLAB). This fit had several constraints: the final solution must place the center within 2 grid points of the seeded fit (parameterized by position and size) and within the mapped visual field; the amplitude must be between 0 and 5 ; the baseline must be between -5 and 5 BOLD $z$ score units. Occasionally, this nonlinear fitting algorithm did not converge and resulted in a larger error than the original seed. In this case, we took the best fit grid point as the final fit.

To test whether vRF fit parameters changed when participants focused spatial attention at different positions, we compared fits during each attend periphery condition with fits during the attend fixation condition. We computed a difference score (attend peripheral - attend fixation) to describe the magnitude of the attentional modulation. For example, a difference score of $-2^{\circ}$ in the FWHM of the vRF would indicate that the response profile width decreased when the participant attended to the peripheral target. This analysis revealed a subset of voxels with very large difference scores, which we determined to be due to noisy data or poor fits via manual inspection. Accordingly, we performed a final thresholding step for all vRF-based analyses: an elimination of outlier voxels with difference scores $>3$ times the SD of the population mean, where the population consists of the parameter difference scores for a given region of interest (ROI) (Table 1). After removing these outliers, we tested whether the vRF parameter difference scores differed significantly from 0 within a visual ROI by bootstrapping the distribution of difference scores across participants 10,000 times.

To determine whether these vRF changes were modulated by their position in the visual field, we first calculated each vRF's distance from the attended location ( $v_{-}$dist_attn) using its position during the fixation task. We then fit an $n$th order polynomial to the vRF difference scores as a function of $v_{-}$dist_attn, where $n=0,1$, or 2 . This corresponds to a constant offset (0th order), a linear fit (first order), or a quadratic or parabolic fit (second order). These fits were cross-validated by fitting on $50 \%$ of the vRF difference scores and calculating goodness-of-fit (residual sum of squares and $R^{2}$ ) on each of the 10,000 cross-validation iterations. These cross-validation iterations also provided CIs on the coefficients for each polynomial. The most parsimonious fit was chosen by performing a nested $F$ test on the average residual sum of squares for each polynomial model.

We also tested whether vRF attentional modulations depended on hemisphere or visual hemifield, akin to the results reported for IPS0-IPS5 in Sheremata and Silver (2015). We sorted the voxels in each attention condition as contralateral or ipsilateral to the attended target. We then performed a series of nonparametric bootstrapped tests similar to a twoway ANOVA with attended hemifield and voxel hemisphere as factors. The vRFs were resampled with replacement across subjects 10,000 times. We then evaluated the two main effects and the interaction by computing a difference in the means of the groups or a difference in the slope between the group means, respectively. None of the tests for the effect of hemisphere and the interaction survived FDR correction, so we do not report those results here. We speculate that this null result is likely due to a lack of reliable voxels in anterior parietal cortex areas IPS1-5 in our study, where previous reports have found larger laterality effects (Sheremata and Silver, 2015).

\section{Population analysis}

Fine spatial discriminability metric. To compute the spatial discriminability of a population of VRFs, we estimated the spatial derivative of each $\mathrm{vRF}$ at every point in the mapped visual field in $0.1^{\circ}$ steps (Fig. $1 c$ ). This was done by taking the slope of the vRF along the $x$ and $y$ direction at each pixel in the image of the visual field and squaring this value (Scolari and Serences, 2009, 2010). This measurement is a descriptor of how well a population code can discriminate small changes in the spatial arrangement of the stimulus array, which depends on the rising and falling edges of a tuning curve rather than the difference between the peak response and a baseline response (Regan and Beverley, 1985; Pouget et al., 2003; Butts and Goldman, 2006; Navalpakkam and Itti, 2007; Scolari and Serences, 2009, 2010). To restrict our measurements to the relevant area near the peripheral target, we computed discriminability values within 1 degree of the center of each target across both spatial dimensions ( $x$ and $y$ ). These were summed and divided by the maximum discriminability value in that population to make the results comparable despite changes in vRF coverage or responsiveness.

Stimulus reconstructions using an inverted spatial encoding model. In addition to computing the discriminability metric described above, we also reconstructed an image of the entire visual field on each trial using a population-level encoding model. Compared with the local spatial discriminability index, this is a more sensitive method of assessing the amount of spatial information encoded in an entire population of voxels because it exploits the pattern of response differences across voxels, rather than treating each voxel as an independent encoding unit (Serences and Saproo, 2012; Sprague et al., 2015).

We trained the spatial encoding model using a procedure similar to the vRF estimation analysis described above (see Fig. 4a). This yields an estimated matrix of weights, $\hat{W}_{2}$, which specifies how much each voxel in a region of interest responds to each of the spatial channels (Brouwer and Heeger, 2009; Serences and Saproo, 2012; Sprague and Serences, 2013; Sprague et al., 2015). We then solved Equation 1 using the MoorePenrose pseudoinverse with no regularization as follows:

$$
\hat{W}_{2}=B C^{T}\left(C C^{T}\right)^{-1}
$$

$C$ was constructed using a set of 54 evenly tiled spatial filters (Eq. 2; 9 horizontal $/ 6$ vertical; spaced $1.25^{\circ}$ apart; $1.56^{\circ}$ FWHM). $\hat{W}_{2}$ was estimated using the data from the jittered position runs. This was done separately for each participant, using a training set balanced across the conditions of interest (e.g., an equal number of attend left and attend right runs and all attend fixation runs because fixation is the neutral condition).

To reconstruct a representation of the mapped visual space, we inverted the model and multiplied the pseudoinverse of the estimated weight matrix $\hat{W}_{2}$ with a test dataset from the discrete position runs $\left(B_{2}\right)$, yielding estimated channel activations for each trial $\left(C_{2} ; k_{2}\right.$ channels by $t$ test trials) (Eq. 6). Thus, we refer to this analysis as the inverted encoding model (IEM) as follows:

$$
\hat{C}_{2}=\left(\hat{W}_{2}^{T} \hat{W}_{2}\right)^{-1} \hat{W}_{2}^{T} B_{2}
$$

Because of mathematical constraints on inverting $W_{2}$ (number of voxels must be greater than number of channels), we included all voxels in each ROI instead of just the subset of well-fit voxels used in the vRF analyses described above. We computed Equation 6 twice using different test datasets, once for the discrete position attend left runs and once for the discrete position attend right runs.

When we multiply the resulting channel activations by a grid of pixels that define the spatial channels, we obtain a spatial representation of the entire visual field on each trial. This image contains a stimulus reconstruction showing where the checkerboard should have been given the trained model and the activation pattern across all voxels in the independent test set. The stimulus reconstructions were then fit in the same manner as the vRFs, using Equation 1 to estimate the center, size, amplitude, and baseline (mean fit RMSE across all ROI reconstructions 0.114; $95 \%$ CI $[0.102,0.312])$. Here, the baseline is an estimate of the multivariate reconstruction that is spatially nonselective (i.e., not significantly modulated by the position of the mapping stimulus). The amplitude 
describes the maximal increase in that reconstruction relative to baseline when the mapping stimulus is on the screen.

To assess how attention changed reconstructions of the mapping stimulus across the visual field, we first computed a difference score that described the effect of attention by folding the visual field in half (i.e., collapsing across hemifield) and comparing parameters in the attended vs ignored hemifield. We excluded the reconstructions that fell along the vertical meridian ( 3 of 51 stimulus positions). This allowed us to control for the overall effect of eccentricity while remaining sensitive to other spatial patterns in stimulus reconstruction modulations.

We then set up a single factor repeated-measures omnibus ANOVA to determine which pairs of ROI and parameter (e.g., V1 size, V1 amplitude) were affected by either attention or Euclidean distance from the target stimuli. The attention factor had two levels (attend/ignore) and the distance factor had 6 levels ( 6 evenly spaced distance bins from $0^{\circ}$ to $\left.2.54^{\circ}\right)$. Based on the results of this omnibus test, we tested any significant ROI-parameter combination in a two-way repeated-measures ANOVA of attention $\times$ distance. To estimate the $p$ values for these tests, we generated empirical null distributions of the $F$ scores by randomizing the labels within each factor 10,000 times within each participant. Reported $p$ values are the percentage of the randomized $F$ scores that are greater than or equal to the real $F$ scores.

Layered spatial encoding model to link $v R F$ s to multivariate stimulus reconstructions. To test how changes in the response properties of the underlying vRFs contributed to changes in the fidelity of region-level stimulus reconstructions, we generated simulated patterns of voxel activity on every trial by predicting the response to each stimulus based on the vRF fit parameters. We then used this simulated data to estimate and invert a populationlevel spatial encoding model, as described above (see Fig. $6 a$ ).

For these simulations, we could only use well-fit voxels to generate simulated BOLD time series. This constrained the analysis to ROIs with at least as many vRFs as spatial filters used to estimate the spatial encoding model. To ensure that we could include most participants in the layered encoding model analysis, we created two large ROIs by merging the smaller retinotopically defined regions described above. The occipital ROI consisted of V1, V2, V3, and V4 defined for each subject. The posterior parietal ROI consisted of V3A/B and IPSO. The vRFs in the parietal ROI show distinct patterns of attentional modulations (see Fig. 2e), suggesting that $\mathrm{V} 3 \mathrm{~A} / \mathrm{B}$ and IPS0 are both anatomically and functionally distinct from the occipital regions (see also de Haas et al., 2014). Although merging ROIs increased the number of voxels available for the encoding model analysis, we still did not have enough voxels in the parietal ROI to estimate the layered encoding model for 3 of the 7 participants (AL, AR, AU). However, the remaining data from 4 participants were sufficient to produce stable subject-averaged results.

To simulate each voxel's BOLD response on every trial that the participant completed in the real experiment, we first created a high-resolution set of spatial channels $\left(21 \times 11\right.$ channels spaced $0.5^{\circ}$ apart, FWHM $=$ $0.65^{\circ}$ ) and generated weights for each channel based on the vRF fit obtained from prior analysis. That is, we evaluated Equation 2 for each channel at the vRF's fit center and adjusted the response gain by multiplying this result by the fit amplitude and adding the fit baseline. We then added independent Gaussian noise to each of these channel weights, simulating a small amount of variance in the voxel's response $(\sigma=0.5)$. Each voxel's channel weights were then multiplied by the idealized channel response on each trial (i.e., the channel filter convolved with the stimulus mask), effectively simulating the BOLD response on each trial for the entire population of voxels based on their measured vRFs. We added Gaussian noise to this simulated response as well $(\sigma=0.5)$. We then computed stimulus reconstructions using the same method as described above (the IEM in Stimulus reconstructions using an inverted spatial encoding model), averaging resulting reconstructions across participants and like positions before fitting.

To ensure the stability of the reconstructions that were based on simulated data, we repeated the simulations 100 times and averaged across the fits of all iterations to generate the plots in Figure $6 b$. Then, to compare how well the layered model reproduced the attentional modulations observed in stimulus reconstructions generated with real data, we calculated an error metric between the layered IEM and the real data. We first calculated reconstruction difference scores across attention condition (attended - ignored; see Stimulus reconstructions using an inverted spatial encoding model). This yielded 24 difference scores each for both attention conditions in both the layered IEM data and the empirical data. Because the empirical data did not have any repeated iterations, we averaged across all 100 iterations of the layered model to match the dimensionality of the real reconstructions $(2$ conditions $\times 24$ difference scores $\times 4$ parameters). We could then calculate the RMSE between the difference scores from the full empirical dataset (i.e., the data shown in Fig. 5) and the modeled data. This was used as a metric to describe the goodness-of-fit of each layered IEM.

We then tested how different vRF attentional modulations contributed to changes in the population-level stimulus reconstructions. To test how shifts in vRF centers contributed to population-level information, we modeled voxels that had the same fit center across both attention conditions, simulated their BOLD responses on each trial, and generated stimulus reconstructions from these data. The voxel's vRF center was defined as the vRF center fit from the neutral attend fixation data. A similar procedure was repeated for all reported combinations of parameter changes across conditions. Again, whichever parameter was held constant took its value from the neutral attend fixation condition.

To calculate the confidence intervals on the RMSE changes in Figure $6 c$, we resampled with replacement across the 100 model iterations and refit the average across these 100 instances. This resampling procedure was repeated 500 times to generate a distribution of fits to the model data. We then took the difference between the RMSE of the null model, in which no parameters varied between attention conditions, and the RMSE of the model which held some number of vRF parameters constant across attention conditions.

\section{Results}

\section{Modulations of vRF properties with spatial attention}

We estimated single vRFs for each voxel in 6 retinotopically identified visual areas from V1 to IPS0. The estimation of vRFs was done independently for each attention condition so that we could compare a single voxel's spatial tuning across conditions.

To confirm that the fit sizes were consistent with previous results, we fit a line to the estimated sizes as a function of the vRF center eccentricity. First, we combined all vRFs across participants and conditions in each ROI. We then binned the vRF centers every $0.25^{\circ}$ from fixation and calculated the mean size (Fig. $2 b)$. We first replicated an increase in vRF size with increasing eccentricity, and an increase in the slope of this relationship across visual regions (Gattass et al., 2005; Dumoulin and Wandell, 2008; Amano et al., 2009; Harvey and Dumoulin, 2011) (Fig. $2 b$ ). These observations confirm that our method produced reasonable vRF estimates that were consistent with previous reports.

Next, we examined how covert attention to the peripheral attention targets modulated $\mathrm{vRF}$ properties, relative to the attend fixation condition. Overall, the center position of vRFs shifted significantly closer to the attended location $(p<0.005$ in all ROIs, Fig. $2 d$ ). This finding is consistent with previous reports in humans and in monkeys for both covert attention tasks and saccade tasks (Womelsdorf et al., 2006, 2008; Klein et al., 2014; Zirnsak et al., 2014).

Although we did observe changes in the size of individual vRFs, the mean change was not significantly different from zero $(p>0.05$ in all ROIs). Size increases have been previously reported in tasks that required subjects to attend to the mapping stimulus, which moved on each trial (Sprague and Serences, 2013; Kay et al., 2015; Sheremata and Silver, 2015). Accordingly, if attention causes the center of RFs to shift toward the attended target, these combined shifts in position would average out to form a larger RF estimate. In contrast, mapping vRFs while maintaining a fixed locus of attention would nullify the size increase, consistent with the results we ob- 
a

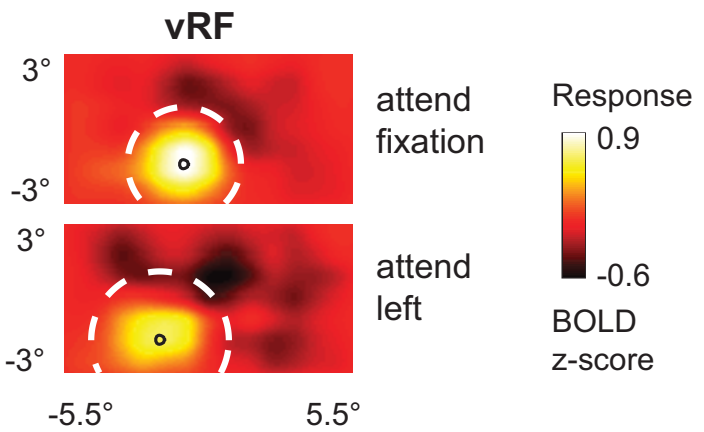

C

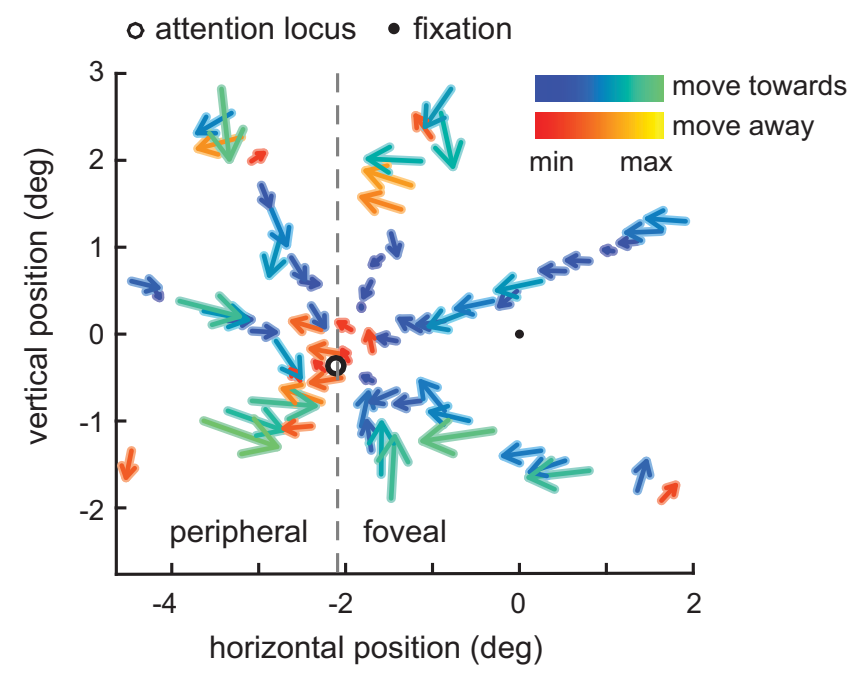

b

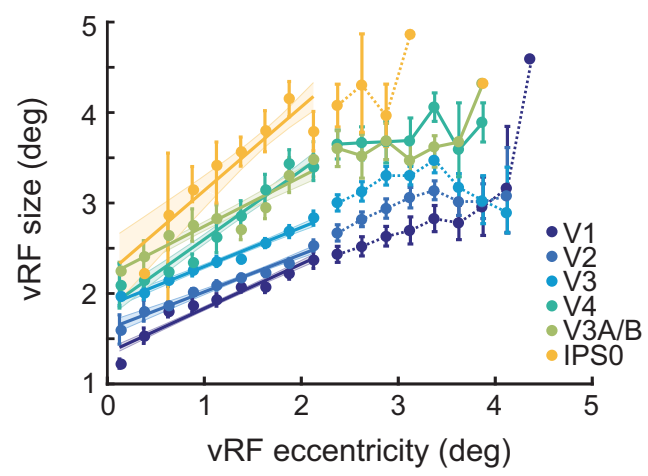

d
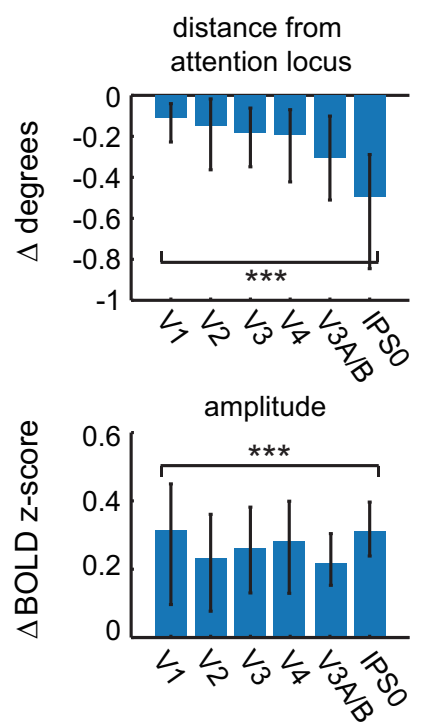

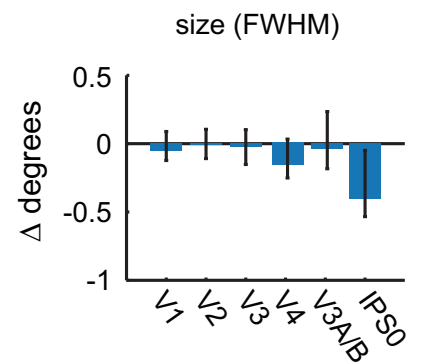

baseline

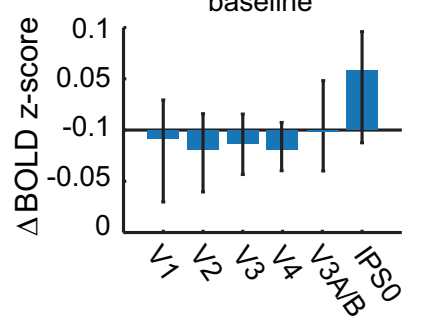

e

$$
\begin{array}{ll}
-\mathrm{V} 1 & -\mathrm{V} 4 \\
-\mathrm{V} 2 & -\mathrm{V} 3 \mathrm{~A} / \mathrm{B} \\
-\mathrm{V} 3 & -\mathrm{IPS} 0
\end{array}
$$

position

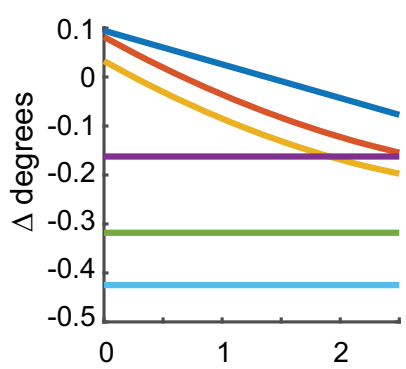

Distance-dependent changes in vRF properties

size

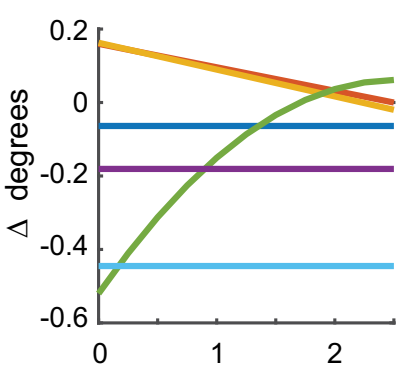

amplitude

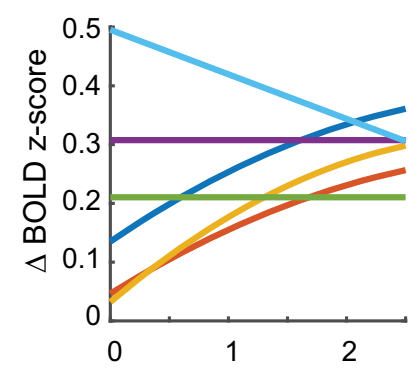

baseline

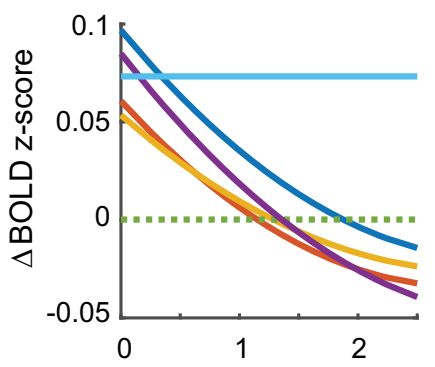

Figure 2. Changes in vRFs across attention conditions. We separately estimated vRFs for every voxel in visual and posterior parietal areas, discarding poorly estimated or noisy voxels (Table 1). Unless otherwise specified, figure data are averaged across subjects. Error bars indicate $95 \%$ Cls computed by resampling the data distribution. $\boldsymbol{a}$, An example vRF shows that attending covertly to the left location shifts the center of the receptive field profile to the left compared with the neutral attend fixation condition. Voxel is from Subject AR in area V3A/B. $\boldsymbol{b}, 0$ ur vRF estimates reproduced the canonical size-eccentricity relationship (positive slope in all ROls, $p<$ minimum possible $p$ value, 1/10,000 iterations) and the increase in slope between visual regions. $c$, Preferred position changes of V4 vRFs with covert spatial attention. We binned each VRF by its position during the attend fixation condition. The origin of each arrow is the center of each position bin. The end of the arrow shows the average position shift of the vRFs within that position bin during the attend peripheral conditions (left/right are collapsed and shown as attend left). The majority of vRFs shift toward the attended location (blue-green color map vs red-yellow). $\boldsymbol{d}$, Mean changes in vRF parameters (attend peripheral target - attend fixation) in each visual area.e, Attentional modulations of each vRF parameter plotted by the vRF's distance from the attention target computed from its position during the attend fixation task (Table 2).

served (Fig. 2d). Another study which also found increases in vRF size with attention required subjects to attend the fixation point while they manipulated the perceptual load, or difficulty, of the attention task (de Haas et al., 2014). In our study, we intentionally kept task performance constant and could not evaluate effects of difficulty on the parameters of vRFs.

We also found an overall increase in vRF amplitude with attention ( $p<0.001$ for all tests). Because these measures were 
Table 2. Mean coefficients for polynomial fits of how vRF parameter change is modulated by distance from the attended location $\left(v \_d i s t \_a t t n\right)^{a}$

\begin{tabular}{llllr}
\hline & Position & Size & Amplitude & \multicolumn{1}{c}{ Baseline } \\
\hline V1 & $-0.069,0.095$ & -0.064 & $-0.019,0.137,0.135$ & $0.011,-0.073,0.010$ \\
V2 & $0.015,-0.133,0.082$ & $-0.064,0.160$ & $-0.016,0.125,0.046$ & $0.011,-0.066,0.061$ \\
V3 & $0.017,-0.135,0.033$ & $-0.073,0.163$ & $-0.025,0.170,0.032$ & $0.009,-0.053,0.054$ \\
V4 & -0.162 & -0.181 & 0.308 & $0.011,-0.078,0.085$ \\
V3A/B & -0.318 & $-0.091,0.461,-0.520$ & 0.210 & $<0.001$ \\
IPS0 & -0.425 & -0.445 & $-0.076,0.495$ & 0.073 \\
\hline
\end{tabular}

${ }^{a}$ Number of reported coefficients in the table correspond to the polynomial order, which has yielded the most parsimonious fit to the data (e.g., 1 coefficient for $n=0,2$ coefficients for $n=1$, etc.).

calculated relative to a fixation task, these data suggest that covert spatial attention to a peripheral location caused widespread position and gain modulations in all vRFs across the visual field.

It is unclear whether these attentional modulations are limited to areas near the attended target, or whether they are uniform across the visual field. For example, vRF position shifts could result in a radial convergence of RFs toward the attended target, or a uniform shift of RFs along a vector extending from fixation to the attention or saccade target (Tolias et al., 2001; Klein et al., 2014; Zirnsak et al., 2014). Furthermore, reports of other RF properties (such as size) modulating with attention have been mixed (Connor et al., 1996, 1997; Womelsdorf et al., 2008; Niebergall et al., 2011; Sprague and Serences, 2013; Klein et al., 2014; de Haas et al., 2014; Kay et al., 2015; Sheremata and Silver, 2015). We therefore examined whether each of the vRF parameter changes was dependent on the vRF's location in the visual field, relative to the attended location. First, we created radial distance bins centered on the left or right attended locations, and sorted voxels into these bins based on their preferred position during the fixation condition. After this sorting procedure, data from the right condition were flipped and collapsed with the left condition.

When we plotted vRF position changes in each bin, we found that spatial attention caused vRF position shifts that converged on the attended location (two-tailed sign test on vector direction, $p<0.001$ in all ROIs). That is, vRFs shifted closer to the attended location (Fig. 2c), compared with when subjects attended fixation (mean shift across all vRFs and ROIs: $-0.239^{\circ}, 95 \%$ CI $[-0.566,-0.048]$, Fig. $\left.2 d\right)$. Small eye movements toward the attended location cannot explain receptive field convergence: this would cause all vRFs to shift in the same horizontal direction, rather than radially converging on one point. These data are consistent with results from both humans (Klein et al., 2014) and macaques (Connor et al., 1996, 1997; Womelsdorf et al., 2006, 2008) that use a similar task. However, the prior study in humans focused only on vRFs with preferred locations that were foveal to the attended location, and the studies in macaques only report RF position changes in V4 and MT. By contrast, our data show that vRF centers converge on the attended location across all visual areas, including primary visual cortex, and that this pattern of modulations includes vRFs peripheral to the attended target.

These plots (Fig. 2a,d) also suggested that vRFs farther from the attended location underwent larger position changes than vRFs near the attended location. That is, the magnitude of the attentional modulation may be dependent on the distance between the vRF and the attended target. To test for this, we fit a polynomial to the vRF parameter changes as a function of distance from the attended location (see Materials and Methods). We selected the most parsimonious fit ranging from a mean change in vRF parameter (0th order polynomial) to a parabolic change (second-order polynomial) by conduct- ing a nested $F$ test (Table 2). The best polynomial fits are plotted in Figure 2e.

This analysis allowed us to characterize trends in vRF attentional modulations across space. It also implicitly tests whether voxels contralateral to the attended target respond differently than ipsilateral voxels. This is because vRFs near the attended target will mostly originate from the contralateral side of visual cortex. Therefore, any fit lines with a significant slope imply there is a difference between contralateral and ipsilateral voxels (Sheremata and Silver, 2015). A separate test described in Materials and Methods confirmed that contralateral voxels differed significantly from ipsilateral voxels in the areas where we saw the highest fit slopes in Figure $2 e$ (FDR-corrected $p<0.05$ for position: V1, V2; size: V3; amplitude: V3, IPS0). However, because the fit lines illustrate how these changes occur over space, we discuss those data here instead (Fig. 2e).

In early visual areas $\mathrm{V} 1-\mathrm{V} 3$, vRFs near the attention target were slightly repelled from the target, whereas vRFs farther from the target were attracted toward the target. In later visual areas, vRFs were uniformly attracted toward the attention target. We saw a similar pattern of results with size modulations: early visual areas showed an increase in vRF size near the attention target, and decreased size farther away. However, in areas V3A/B and IPS0, vRF size decreased near the attention target.

The pattern of vRF amplitude modulations was also segregated between the early and later visual areas. All vRFs increased in amplitude with attention, but the slope of this relationship inverted from early to later visual areas. In V1-V3, the slope if positive, such that voxels $\sim 2^{\circ}$ away from the attention target increase in amplitude more than voxels right at the target position. The amplitude increase is constant in V4 and V3A/B. Finally, in IPS0, the slope inverts to become negative, so that voxels near the attention target increase in amplitude more than voxels farther away. Last, we found an increase in vRF baseline near the attended target in V1-V4, but a uniform increase in baseline in IPS0. Overall, we found that the type and magnitude of the attentional modulation in different visual areas changes as a function of the spatial relationship between vRFs and the attended target. This is consistent with findings from macaque neurophysiology, which had suggested that amplitude and size changes depend on where the RF is located in relation to the attended target (Connor et al., 1996; Niebergall et al., 2011).

These fits only describe the modulations with respect to the voxel's position during the attend fixation task. However, these parameter changes likely interact with one another, such that a voxel that shifts toward the attended location will also increase in amplitude. Hence, to determine how the joint patterns of vRF modulations change the spatial information content of a representation, in the next section we discuss two different population-level measures that combine data across the population of vRFs in each ROI. 
a

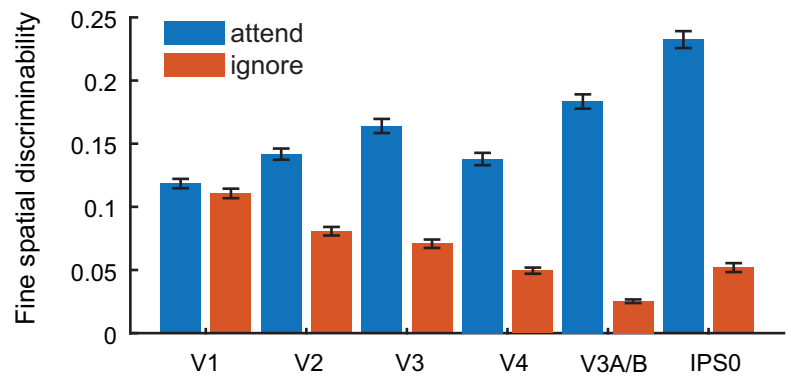

b

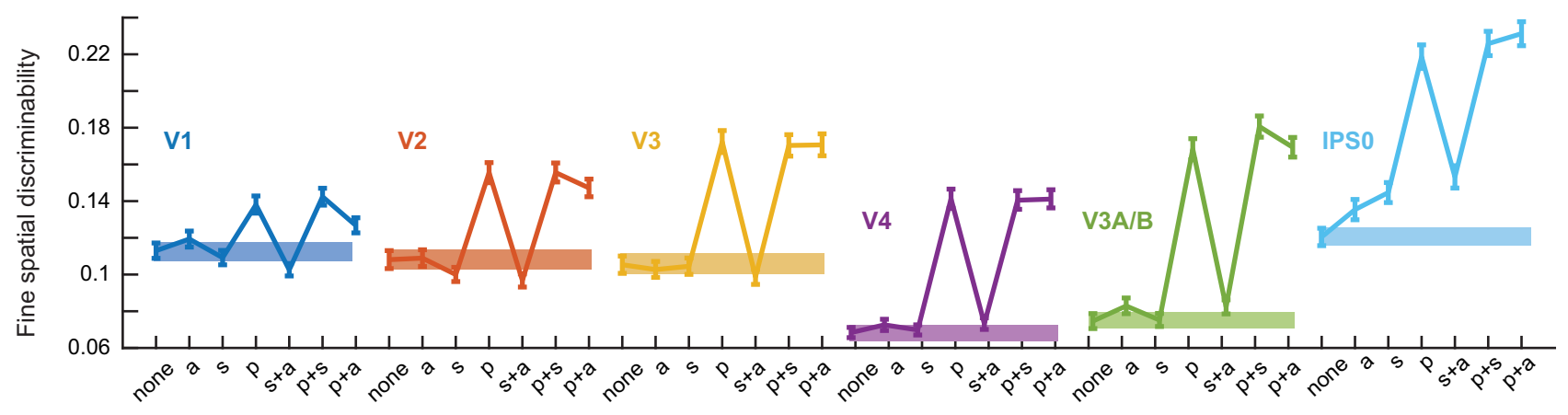

Figure 3. Spatial discriminability increases with attention and is mediated by position changes in vRFs. Error bars indicate bootstrapped 95\% Cls. a, We formulated a measurement to describe the ability of a population of voxels to make fine spatial discriminations around the attention target. We used the properties of each voxel's spatial tuning curve to make this measurement (see Materials and Methods). Spatial discriminability increased when subjects attended the target, compared with when they ignored the target in the opposite hemifield (resampled $p<$ minimum possible $p$ value $1 / 1000$ for all ROIs). $\boldsymbol{b}$, The discriminability metric was recomputed for vRFs with a variety of attentional modulations. Abscissa labels from left to right: None, vRF parameters during the neural attend fixation condition; a, amplitude; $s$, size; $p$, position. Spatial discriminability increased significantly when we applied position changes measured during the attend L/R task to the vRFs compared with when we applied no parameter changes (solid bar). By contrast, applying size changes did not change spatial discriminability in most ROls, although it did cause a small increase in IPSO.

\section{Increases in spatial discriminability depend primarily on vRF position shifts}

Next, we assessed how different types of RF modulations influenced the precision of population-level codes for spatial position. We first computed a discriminability metric that described the ability of a population of tuning curves (here, vRFs) to support fine spatial judgments (see Materials and Methods). When we computed this metric based on the measured vRF properties from each condition, spatial discriminability near the attended target increased relative to the ignored target in the opposite visual hemifield (Fig. 3a).

We then tested how different types of vRF modulations (such as size changes or position shifts) affected this spatial discriminability metric. As a baseline comparison, we first computed discriminability based on vRFs estimated during the attend fixation runs for each subject. We then added different sets of observed attentional modulations to the population before recomputing spatial discriminability. For example, we shifted all the vRF centers to match the measurements when a subject was attending to the left target and computed discriminability near the attended target. Because the response baseline of a vRF does not affect the discriminability metric, we excluded this type of attentional modulation from these analyses.

Across all ROIs, we found that vRF position shifts played the biggest role in increasing fine spatial discriminability compared with changes in size or changes in amplitude (Fig. $3 b$ ). Position modulations alone led to a large increase in spatial discriminability, whereas other combinations of parameter modulations only had an impact if we added in position shifts (i.e., a change in size and position increased discriminability, but size alone did not).
The only departure from these patterns was observed in IPS0, where all attentional modulation types increased spatial discriminability, but position changes increased spatial discriminability the most.

\section{Spatial attention increases the fidelity of population-level stimulus reconstructions}

By design, the spatial discriminability metric we computed is only informative about local spatial representations and cannot assess how different patterns of vRF modulations might result in representational changes across the visual field. To address this point, we built a multivariate spatial encoding model to measure how attention changes the representations of visual information in disparate parts of space. This also allowed us to further test the effects of vRF modulations on the encoding properties of the population, including response baseline changes that were not captured by our discriminability metric.

The spatial IEM reconstructed an image of the entire visual field on each test trial. We first trained the model using the responses of each voxel on a set of training trials with known mapping stimulus positions. We then created image reconstructions on independent test trials by inverting the model and multiplying it by the voxel responses during each test trial (Fig. $4 a$; see Materials and Methods). Each image contained a representation of where the mapping stimulus should have been given the pattern of voxel activations on that particular trial. The IEM successfully reconstructed the task-irrelevant mapping stimuli using activation patterns across voxels in each visual area from V1 through IPSO (Fig. $4 b$; grand mean error between fit and actual position $2.40^{\circ}, 95 \%$ CI $\left.0.55^{\circ}, 4.97^{\circ}\right)$. 


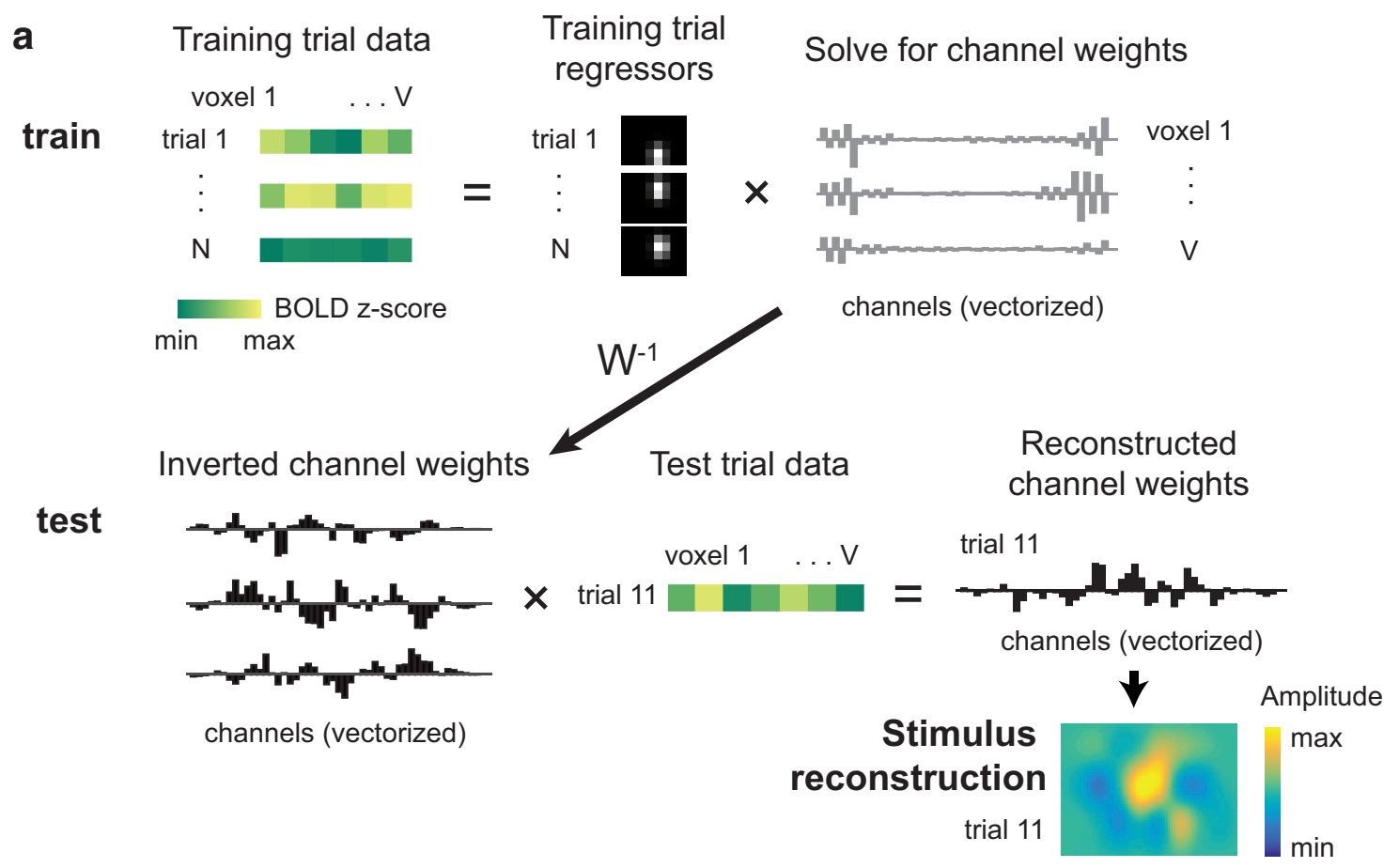

b
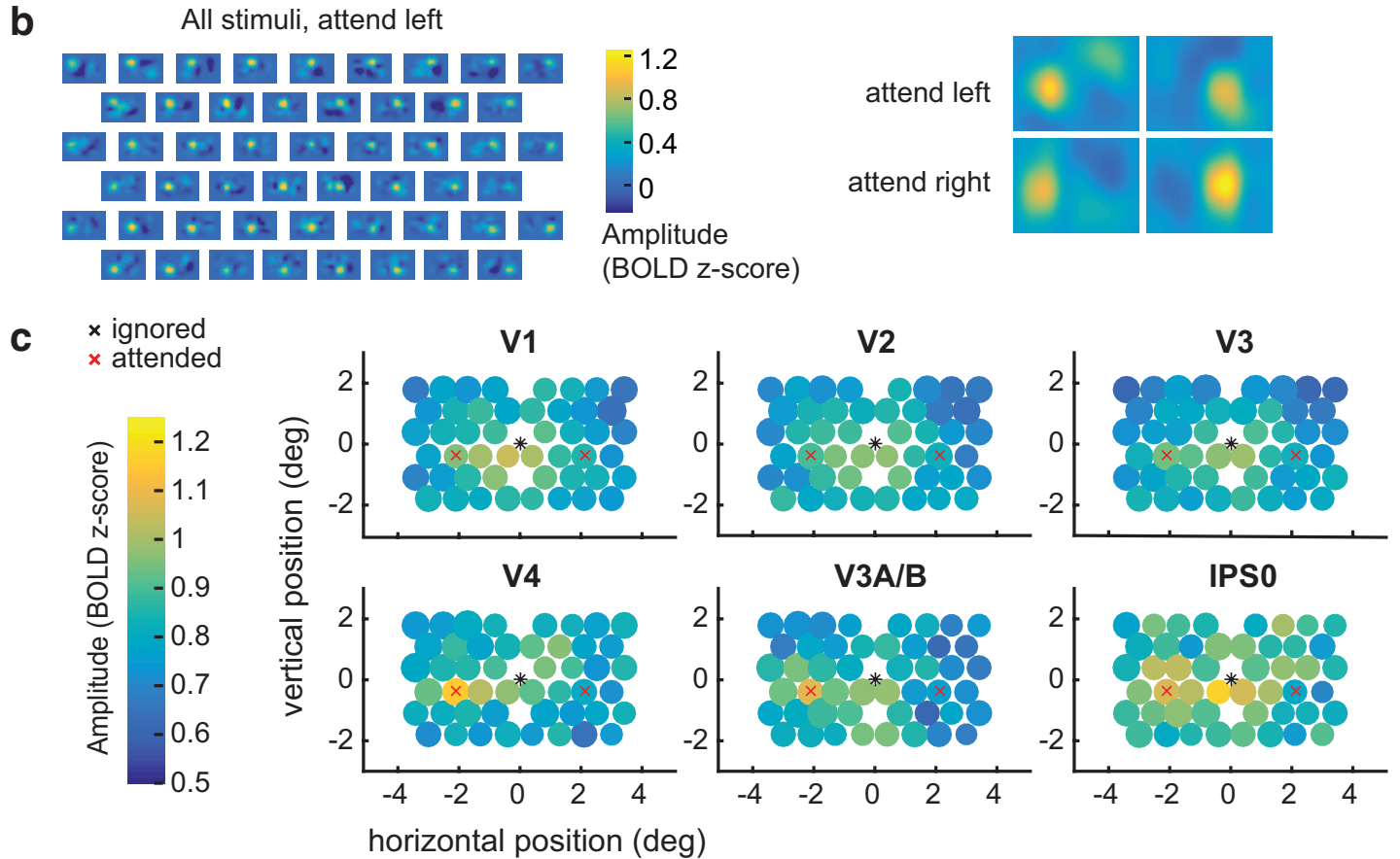

horizontal position (deg)

Figure 4. Multivariate IEM used to reconstruct the mapping probe stimuli. $\boldsymbol{a}$, To train the IEM, we first take the BOLD data from all voxels within a visual region from a subset of training trials. Then, we solve for a set of channel weights using least-squares regression. To reconstruct the stimulus, we invert this weight matrix and multiply it with BOLD data from the same voxels during a test trial. This yields a reconstructed channel response profile, which can be transformed into a reconstruction of the mapping stimulus on every trial in each attention condition. Data shown are examples from Participant AR for a subset of V1 voxels. $\boldsymbol{b}$, Example stimulus reconstructions for Participant Al in area V1. These reconstructions were averaged across trials with the same position, yielding 51 reconstructions: one for each unique position in the test dataset. Left, The same averaged position reconstructions are shown for each condition. The amplitude on the left is higher when attending left, and on the right when attending right. c, Average reconstruction sizes and amplitudes for each stimulus position (collapsed across condition; left, attended). The diameter of the circle depicts the average fit FWHM of the reconstructions at that spatial position. Reconstruction amplitude was greater in the attended hemifield compared with the ignored hemifield in areas V3A/B and V4 $(p<0.005 ;$ Table 3; Fig. 5).

We used these stimulus reconstructions as a proxy for the quality of the spatial representations encoded in a population of voxels. This is line with previous studies showing that stimulus reconstructions change in amplitude or size as a function of cognitive demands. (Brouwer and Heeger, 2013; Ester et al.,
2013; Sprague and Serences, 2013; Sprague et al., 2014, 2015, 2016).

First, we compared how reconstructed representations of each mapping stimulus changed as subjects shifted their spatial attention. We ran a repeated-measures ANOVA of attention $\times$ dis- 

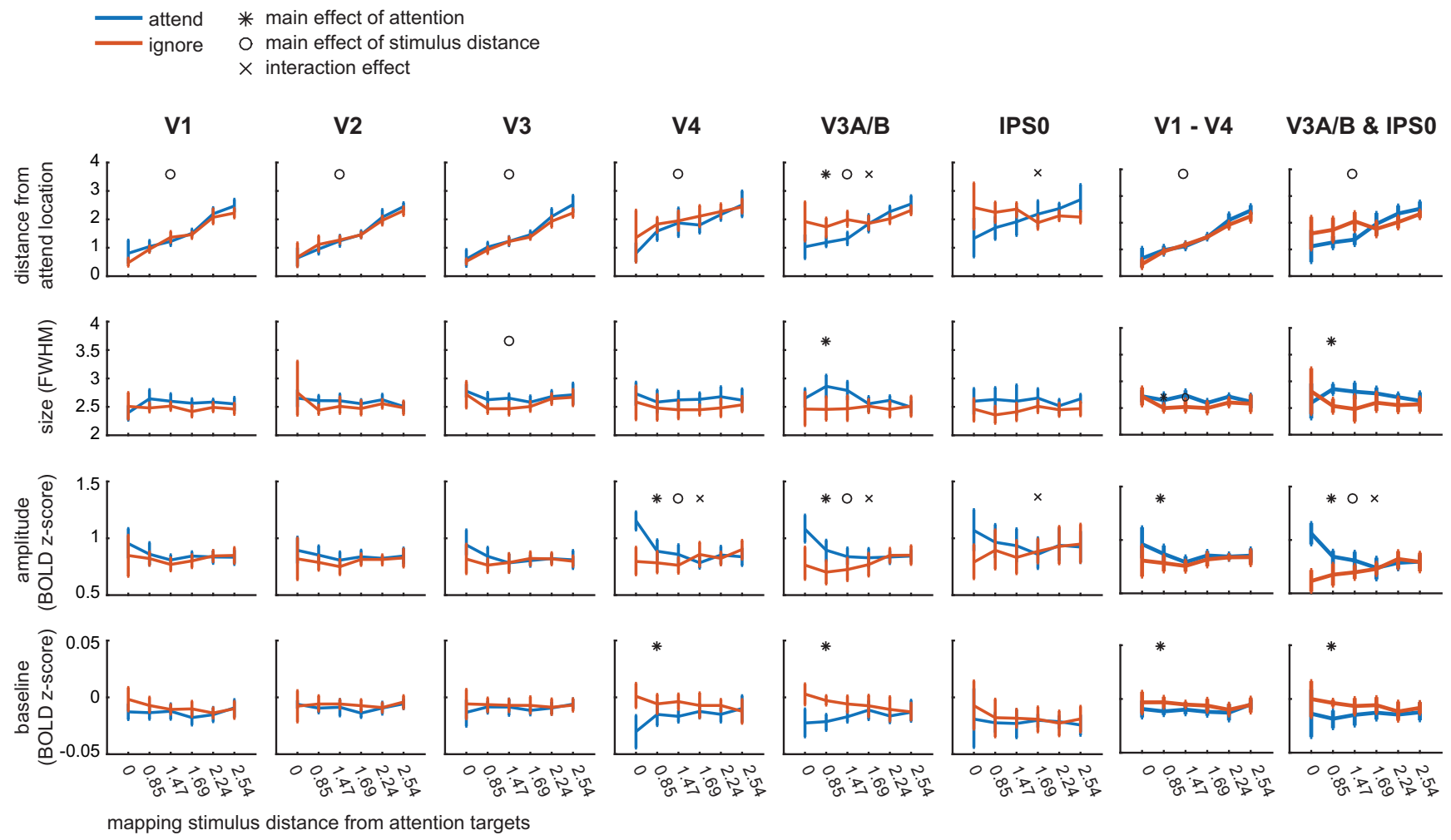

Figure 5. Reconstruction parameters as a function of mapping stimulus distance from the covertly attended locations and attention hemifield (attended vs ignored). For complete list of $p$ values, see Table 3.

Table 3. Two-way ANOVA results for reconstruction parameter changes (s_dist_attn $\times$ attention hemifield)

\begin{tabular}{|c|c|c|c|c|c|c|c|c|}
\hline & V1 & V2 & V3 & V4 & V3A/B & IPSO & V1-V4 & V3A/B and IPSO \\
\hline \multicolumn{9}{|l|}{ Omnibus test } \\
\hline Position & $<0.001^{a}$ & $<0.001^{a}$ & $<0.001^{a}$ & $<0.001^{a}$ & $<0.001^{a}$ & $0.001^{a}$ & $<0.001^{a}$ & $<0.001^{a}$ \\
\hline Size & 0.216 & 0.565 & 0.019 & 0.428 & $0.006^{a}$ & 0.121 & $0.001^{a}$ & 0.110 \\
\hline Amplitude & 0.174 & 0.579 & $0.024^{a}$ & $<0.001^{a}$ & $<0.001^{a}$ & $0.008^{a}$ & $0.016^{a}$ & $<0.001^{a}$ \\
\hline Baseline & 0.088 & 0.734 & 0.934 & $<0.001^{a}$ & $0.001^{a}$ & 0.937 & $0.015^{a}$ & 0.241 \\
\hline \multicolumn{9}{|c|}{ Main effect of distance } \\
\hline Position & $<0.001^{a}$ & $<0.001^{a}$ & $<0.001^{a}$ & $<0.001^{a}$ & $<0.001^{a}$ & 0.192 & $<0.001^{a}$ & $<0.001^{a}$ \\
\hline Size & - & - & - & - & 0.484 & - & $0.019^{a}$ & - \\
\hline Amplitude & - & - & 0.140 & $0.002^{a}$ & $0.005^{a}$ & 0.478 & 0.100 & $0.002^{a}$ \\
\hline Baseline & - & - & - & 0.829 & 0.916 & - & 0.210 & - \\
\hline \multicolumn{9}{|c|}{ Main effect of attention } \\
\hline Position & 0.371 & 0.916 & 0.346 & 0.067 & $0.005^{a}$ & 0.254 & 0.401 & 0.343 \\
\hline Size & - & - & - & - & $0.003^{a}$ & - & $0.005^{a}$ & - \\
\hline Amplitude & - & - & 0.069 & $<0.001^{a}$ & $0.005^{a}$ & 0.158 & $0.049^{a}$ & 0.004 \\
\hline Baseline & - & - & - & $0.001^{a}$ & $<0.001^{a}$ & - & $0.004^{a}$ & - \\
\hline \multicolumn{9}{|c|}{ Interaction of distance and attention } \\
\hline Position & 0.052 & 0.588 & 0.541 & 0.657 & $<0.001^{a}$ & $<0.001^{a}$ & 0.121 & 0.026 \\
\hline Size & - & - & - & - & 0.077 & - & 0.271 & - \\
\hline Amplitude & - & - & 0.064 & $<0.001^{a}$ & $<0.001^{a}$ & $0.004^{a}$ & 0.224 & $<0.001^{a}$ \\
\hline Baseline & - & - & - & 0.019 & 0.011 & - & 0.370 & - \\
\hline
\end{tabular}

${ }^{a_{p}}$ value passed FDR correction ( $q=0.05$, corrected across ROIs and comparisons within each parameter).

tance bin for each reconstruction fit parameter (see Materials and Methods). Here, a main effect of attention would suggest that stimulus reconstructions in the attended hemifield changed in a consistent way compared with the ignored hemifield. A main effect of distance would suggest that stimulus reconstruction changes had a consistent spatial pattern across both the attended and ignored hemifields. This would occur when a stimulus' representation was altered with distance from the attention target. For example, the stimulus reconstruction center should vary lin- early with the stimulus' true distance from the attention target. And last, an interaction effect would suggest that the distance effect was dependent on whether the reconstruction belonged to the attended or ignored hemifield. In our task, the reconstructed stimuli are always irrelevant to the task of the observer. We therefore predicted an interaction effect where spatial attention would selectively modulate stimulus reconstructions within the hemifield of the attended location, but not the opposite hemifield (Connor et al., 1996, 1997). 
a

\section{Layer 2}

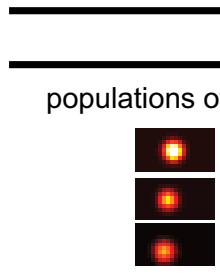 \\ Layer 1}

invert spatial encoding model test trials (attend fixation)
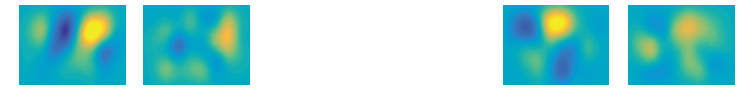

enerated stimulus reconstructions

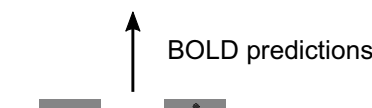

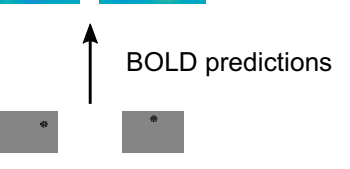

\section{train multivariate spatial encoding model}

Combined V1 - V4

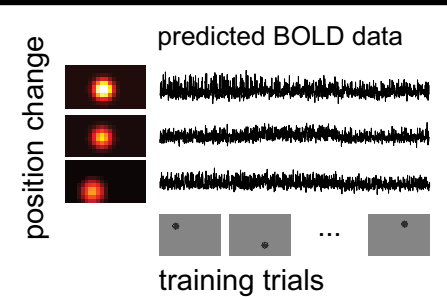

predicted BOLD data

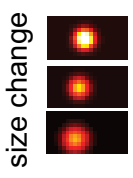

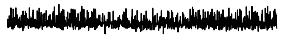

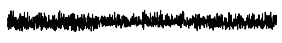

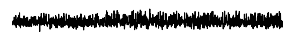

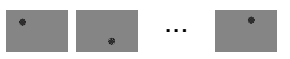

training trials

b

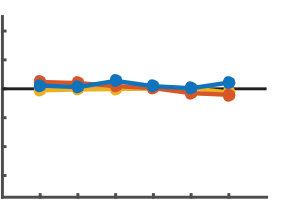

Combined V3A/B \& IPS0

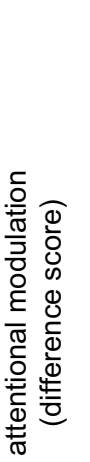
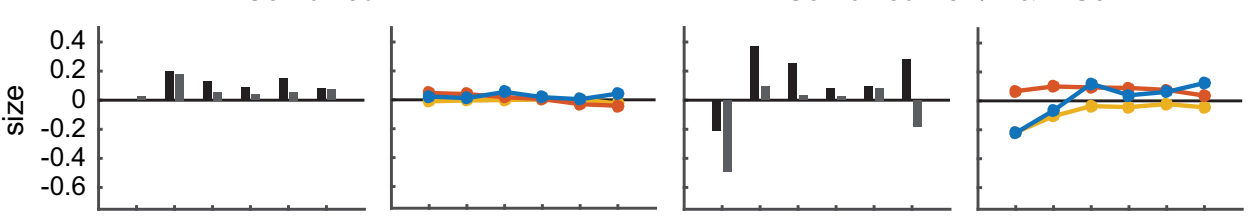

IEM real data

IEM reduced data
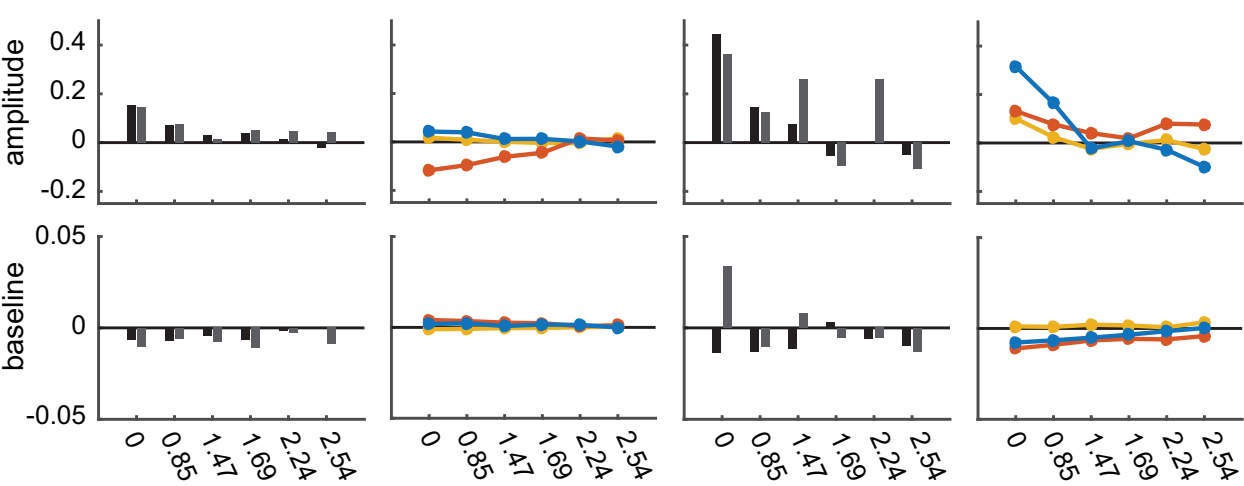

distance from attention target

C

Combined V1 - V4

Combined V3A/B \& IPS0
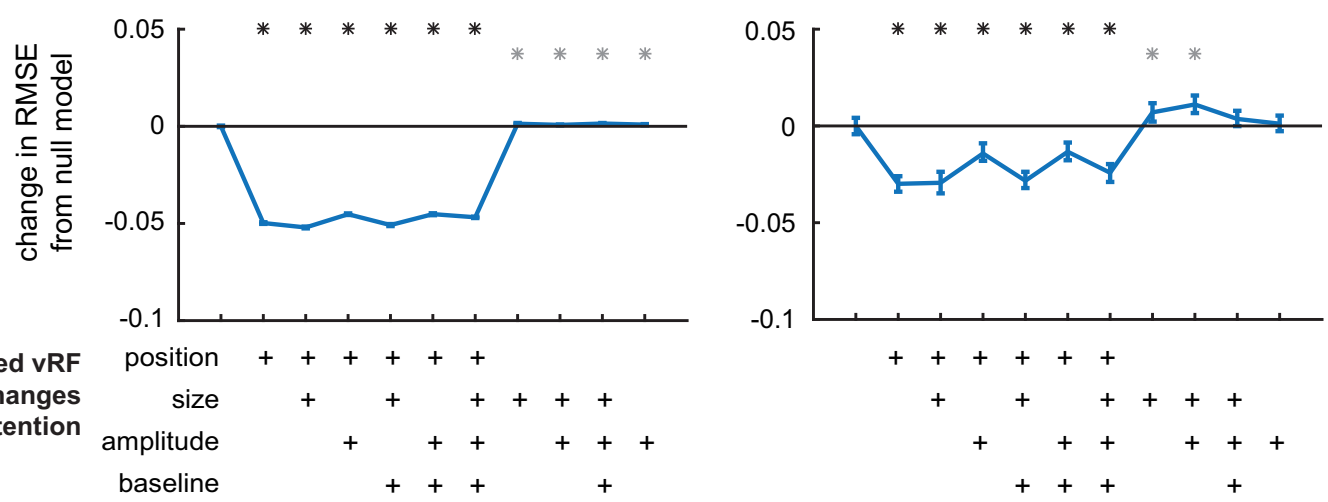

Figure 6. A layered spatial encoding model reveals how different sets of vRF changes lead to enhancements in multivariate stimulus reconstructions. $\boldsymbol{a}$, The first layer of the model uses the vRF fits to generate BOLD data from every subject's real trial sequence. Then the BOLD data from all voxels within one ROI are used to train a multivariate spatial encoding model and reconstruct the mapping stimuli. $\boldsymbol{b}$, Change in reconstruction amplitude in the attended versus the ignored hemifield. We only show reconstruction parameters with significant attentional modulations in the prior IEM analysis (Fig. 5; Table 3). Stimulus reconstructions computed with a reduced number of voxels (gray bar) largely reproduce the pattern of attentional modulations observed in IEMs computed with all voxels (black bar). Furthermore, a comparison of layered IEMs using simulated data revealed that vRF position changes (blue lines) in the first layer of the model are better at reproducing the amplitude modulations in the stimulus reconstructions of the parietal ROI than models that simulate changes in vRF size or amplitude (yellow and red lines). $c$, RMSE between each set of IEM fits and the full empirical dataset fits shown in Figure 5. The null baseline model (far left) is a layered IEM where the vRF parameters are the same across all attention conditions. We then added vRF attentional modulations for each parameter as shown in the matrix below the plot, where all models with position changes are on the left side. ${ }^{*} p<0.05$ (FDR-corrected), models differed significantly from the null baseline model. Gray asterisks indicate an increase in RMSE from the null model. Black asterisks indicate a decrease. 
Table 4. RMSE (and 95\% Cls) between reconstructions from the reduced dataset (only using voxels with RFs) or from different versions of the layered IEM using the same voxels ${ }^{a}$

\begin{tabular}{|c|c|c|c|c|c|c|}
\hline & Reduced data & $\mathrm{p} / \mathrm{s} / \mathrm{a} / \mathrm{b}$ & $\mathrm{p} / \mathrm{a} / \mathrm{b}$ & $\mathrm{p} / \mathrm{s} / \mathrm{b}$ & $\mathrm{s} / \mathrm{a} / \mathrm{b}$ & $\mathrm{p} / \mathrm{a}$ \\
\hline Combined occipital V1-V4 & $0.133(0.109,0.170)$ & $0.146(0.146,0.146)$ & $0.148(0.147,0.148)$ & $0.142(0.141,0.143)$ & $0.194(0.194,0.194)$ & $0.148(0.148,0.148)$ \\
\hline Combined parietal V3A/B and IPSO & $0.834(0.744,0.959)$ & $0.415(0.410,0.419)$ & $0.426(0.422,0.431)$ & $0.411(0.407,0.416)$ & $0.443(0.439,0.447)$ & $0.425(0.421,0.430)$ \\
\hline
\end{tabular}

${ }^{a}$ To generate Cls, the resampling of the real data is performed at the level of the fits to the reconstructions, whereas resampling layered IEM RMSEs is described in Materials and Methods. Abbreviations for vRF changes included in each model: None, vRF parameters identical to fixation condition; $a$, amplitude; $s$, size; $p$, position; $b$.

We found that reconstruction amplitude was selectively increased near the attended location in V4, V3A/B, and IPSO (interaction effect, bootstrapped $p<0.005$; Fig. 5; Table 3 ). This can be interpreted as a local boost in signal-to-noise ratio. Prior reports found that attending to the mapping stimulus, as opposed to attending to a peripheral target in the current experiment, caused an increase in the amplitude of all stimulus reconstructions (Sprague and Serences, 2013). That is, representations of task-relevant stimuli increased in signal-to-noise ratio. We find here that even representations of task-irrelevant stimuli near the attended region of space increase in amplitude, consistent with the idea of an attentional "spotlight," which boosts the fidelity of spatial representations near the attention target.

Although the amplitude interaction effect was present in most visual areas we tested (Fig. 5), we found other effects limited to $\mathrm{V} 3 \mathrm{~A} / \mathrm{B}$ and IPS0 that involved modulations in stimulus representations in the ignored hemifield. In these regions, we found that stimulus reconstructions in the ignored hemifield shifted away from the ignored target location (interaction, bootstrapped $p<$ $0.005)$. We also observed a relative size increase near the ignored attention stimulus in IPS0 (interaction, bootstrapped $p<0.005$ ). These results suggest that stimulus reconstructions in the ignored hemifield are less spatially precise in posterior parietal cortex. Finally, there was also a main effect of attention on reconstruction size and baseline in areas V3, V4, and V3A/B (bootstrapped $p<0.005$ ). However, unlike the interaction effect in IPS0, these size and baseline changes did not vary as a function of distance between the reconstruction and the attended target location.

\section{Using a layered encoding model to explore how single vRFs change population-level codes}

In our final analysis, we used a layered spatial encoding model to determine how changes in vRF properties affected the representations of mapping stimuli in the multivariate reconstructions discussed in the previous section (Figs. $1 c, 4 a$ ). The goal of this analysis was to determine which vRF modulations contribute the most to the observed increase in the amplitude of stimulus representations around the attended location (Fig. 5). This analysis thus complements our analysis of the spatial discriminability metric, which demonstrated that vRF position changes significantly increased the ability of the population to make fine spatial discriminations near the attention target (Fig. $3 b$ ).

The layered spatial encoding model we built links the response properties of single voxels to the encoding properties of a whole population of voxels in a region of visual cortex (Fig. $6 a$ ). In the first layer of the model, we used the fit vRFs to generate simulated BOLD data from each voxel under different attention conditions. We then repeated the multivoxel stimulus reconstruction analysis on this simulated data to model population results for the second layer of the model. This approach allowed us to perform virtual experiments to test how changes in the first layer impacted the second layer. That is, we manipulated which vRF parameters changed with attention (first layer) and observed the resulting changes in the population-based stimulus reconstructions (sec- ond layer). For example, we could test whether an overall increase in $v R F$ response gain with attention would be necessary or sufficient to reproduce the amplitude increases observed in the empirical stimulus reconstructions reported in Figure 5. These virtual experiments also allowed us to compare the relative impact of one type of response modulation (e.g., size changes) with other types of response modulations (e.g., position shifts).

Because the population-level stimulus reconstructions require many voxels from each subject to produce stable and reliable results, we combined the data across several regions in each individual subject before estimating the IEM (for a longer discussion, see Materials and Methods). This yielded one occipital region that combined data from areas V1, V2, V3, and V4, and one posterior parietal region that combined data from V3A/B and IPSO. We repeated the IEM analysis described in the previous section on these larger regions and found that the pattern of attentional modulations observed earlier was consistent in the large ROIs (Fig. 5). Next, to verify whether we could perform the layered IEM using a reduced number of voxels, we reran the IEM analysis but only used the data from voxels with well-fit vRFs. The reduced dataset with fewer voxels reproduced the main pattern of results we observed in the previous section. In particular, covert attention led to an increase in the amplitude of reconstructions near the locus of attention (Fig. $6 b$, black vs gray bars).

We then investigated the contribution of each vRF parameter to the population-level stimulus reconstructions, in a comparison akin to the spatial discriminability analysis in Figure 3. A model that only simulated the observed vRF amplitude or vRF size modulations did not predict the observed increase in reconstruction amplitude near the attention target (Fig. 6b, red lines). However, a layered model that only simulated vRF position changes did predict a large increase in reconstruction amplitude near the attention target in the parietal ROI (Fig. $6 b$, blue line on right). This is consistent with the effects observed in the full dataset (Fig. 5; Table 3), where we only observed an interaction of stimulus distance and attention in the parietal ROI.

To more formally quantify each manipulation of the layered IEM, we calculated an error metric to describe how well each model reproduced the attentional modulations in the empirical data (using the RMSE) (Table 4). We compared each model's RMSE with a baseline model, which did not simulate any vRF attentional modulations (Fig. $6 c$, far left). This null baseline should have the highest error, and any good models should decrease the RMSE between the simulated data and the empirical data. Conversely, a model with higher RMSE is worse at accounting for the empirical data compared with the null model. In both the occipital and parietal ROIs, adding vRF position shifts to the layered model decreased RMSE while abolishing position shifts generally increased the model error (Fig. $6 c$ ). These data are consistent with the results from the spatial discriminability analysis. Together, they suggest that shifts in vRF position have the largest impact on population-level representations, whereas changes in vRF size or gain play smaller roles in changing the fidelity of the population code. 


\begin{tabular}{llllll}
\hline $\mathrm{s} / \mathrm{a}$ & $\mathrm{p} / \mathrm{s}$ & $\mathrm{p}$ & $\mathrm{d}$ & $\mathrm{s}$ & None \\
\hline $0.194(0.194,0.194)$ & $0.141(0.141,0.141)$ & $0.143(0.143,0.143)$ & $0.194(0.194,0.194)$ & $0.194(0.194,0.194)$ & $0.193(0.193,0.193)$ \\
$0.451(0.447,0.456)$ & $0.410(0.405,0.416)$ & $0.410(0.406,0.414)$ & $0.441(0.437,0.445)$ & $0.447(0.442,0.452)$ & $0.834(0.744,0.959)$ \\
\hline
\end{tabular}

\section{Discussion}

By simultaneously measuring the response properties of both single voxels and populations of voxels within retinotopic areas of visual cortex, we could link attentional modulations of spatial encoding properties across scales. Our data provide an initial account of how different types of RF modulations improve the quality of population codes for visual space. First, we show how $\mathrm{vRF}$ attentional modulations depended on the distance between the vRF's preferred position and the static attention target (Fig. 2 ). We then found that shifts in the preferred position of vRFs near the attended target increased the spatial discrimination capacity of a population of voxels (Fig. 3), as well as the amplitude of stimulus reconstructions based on response patterns across all voxels in a ROI (Figs. 5, 6).

\section{Attentional modulations of spatial RFs}

We provide new data on how vRF responses are modulated around a covertly attended static target (Sprague and Serences, 2013; Klein et al., 2014; de Haas et al., 2014; Kay et al., 2015; Sheremata and Silver, 2015). Like prior macaque studies, we find that vRF position shifts depend on the vRF's distance from the attended target (Connor et al., 1996, 1997). However, we also found that the pattern of attentional modulations differs across the visual hierarchy. In V4, V3A/B, and IPS0, voxels shift toward the attended target, whereas in earlier areas, vRFs near the attended target are slightly repelled from it (Fig. 2e). We also found distinct patterns of size modulations: vRF size increased near the attention target in early visual areas but decreased in parietal areas V3A/B and IPS0. Comparison with the existing literature suggests that patterns of RF size modulations likely depend on the nature of the spatial attention task. In fMRI tasks where subjects attended to the mapping stimulus, rather than a static position, researchers report that average $\mathrm{vRF}$ size increases with attention (Sprague and Serences, 2013; Kay et al., 2015; Sheremata and Silver, 2015). RFs in macaque area MT shrink when measured with a mapping probe smaller than the stimulus but increase in size when macaques track the mapping probes as they move across the screen (Womelsdorf et al., 2006, 2008; Anton-Erxleben et al., 2009; Niebergall et al., 2011). This may be because the RFs shift position to track the probe, causing an apparent increase in overall size. Last, manipulating perceptual load at fixation also increases vRF size in human visual cortex (de Haas et al., 2014). Together, these observations demonstrate that the pattern of RF response modulations depends both on task demands and on the spatial relationship between the attended target and the encoding unit's RF.

We note that, although the similarity between attentional modulations of single cell RFs and single vRFs is compelling, their properties are derived from different input signals and are not interchangeable. fMRI voxels in retinotopically organized regions of visual cortex sample from a broad array of neurons with approximately the same spatial tuning preferences, so a position shift in a vRF could either be driven by a change in the preferred position of single neurons, or by a change in the gain profile across neurons tuned to slightly different locations in the visual field. Similarly, single neuron RFs receive input from smaller RFs in earlier visual areas, and a position shift could arise from either mechanism described above (McAdams and Maunsell, 1999; Baruch and Yeshurun, 2014; Dhruv and Carandini, 2014). Because of this inherent ambiguity when measuring the encoding properties of a locally tuned unit, it is useful to compare them with attentional modulations of populationlevel representations.

\section{Attention boosts the spatial encoding fidelity of a population}

We first measured the overall capacity of a population of voxels to make fine spatial discriminations in a region of space. We found that attention increased spatial discriminability near the attended target, relative to the ignored target. We then performed virtual experiments on the vRFs contributing to the population to determine how they affected the spatial discriminability metric. We report that $\mathrm{VRF}$ position shifts increased spatial discriminability significantly more than vRF size changes or gain changes (Fig. 3).

Because the spatial discriminability metric (Fig. 3) is only informative about a local portion of space, we performed a second population analysis to reconstruct an image of the entire visual field on each trial using a multivariate IEM. Attention increased the amplitude of stimulus reconstructions near the attention target, indicating an increase in representational fidelity that accompanied the increase in spatial discriminability. In addition, a layered spatial encoding model revealed that shifts in vRF position could account for these attentional enhancements in the population-level stimulus reconstructions, but changes in vRF size could not. Together, our data demonstrate that shifts in position of many RFs may be a dominant way that single encoding units alter the properties of a population spatial code.

Although population-level information increased the most with changes in vRF position, we reiterate that these position changes could arise from spatially specific patterns of gain modulations in input RFs. If this is true, it is possible that gain modulations with attention may exert their largest effects on the downstream population, where these patterns of gain changes become apparent shifts in vRF position. However, this remains an open question for future work to address.

Our findings also underscore the fact that changes in the spatial encoding properties of single units do not directly translate into analogous changes in the encoding properties of a population of those same units. For example, an overall change in vRF size does not necessarily change the size of the population-level representation (Sprague and Serences, 2013; Kay et al., 2015). Although we found that single units shifted their preferred position toward the attended target, population-level representations did not generally shift with attention. When the population code did shift its encoded position, we found that it was typically representations of the ignored stimulus that shifted farther from the true stimulus location (Fig. 5), consistent with more error-prone representations of irrelevant stimuli. These types of differences further emphasize the need to understand the effects of cognitive state on population codes for the entire visual scene, rather than focusing solely on single units. 
Last, we note that our population-level data do not address the open question of whether RF attentional modulations have perceptual consequences because it is not clear how the spatial encoding models measured here are linked to visual perception and behavior (Koenderink, 1990; Rose, 1999; Anton-Erxleben and Carrasco, 2013; Klein et al., 2016). Further investigation into these topics should include task manipulations to investigate how attentional modulations of both vRFs and population-level metrics track psychophysical performance.

\section{Tuning shifts and labeled lines}

Historically, shifts in the tuning of a RF have not been considered one of the main mechanisms by which attention modulates population-level information, although recent reports suggest that this view is being reconsidered (David et al., 2008; AntonErxleben and Carrasco, 2013). This may be due to "labeled-line" theories of visual information processing, which posit that a single neuron has a consistent feature label, which downstream neurons rely on to perform computations and transmit stable information (Barlow, 1972; Doetsch, 2000; David et al., 2008). When a spatial RF shifts position as a function of cognitive state (e.g., attention), that single neuron's feature label is no longer consistent. Without an accompanying shift in the downstream neurons receiving the changing feature label, such a change could disrupt the stability of the population code. However, our results suggest that population-level spatial representations remain relatively stable, and are even enhanced, when the tuning of the underlying vRFs shift in position, size, and gain.

An alternate proposal to a labeled line code relies on the joint information encoded across a population of cells (Erickson, 1982; Doetsch, 2000). This may occur at several scales; for example, V2 could use the pattern of information from V1 inputs to form a visual representation. This idea is more akin to an encoder-decoder model in which the downstream decoder does not need information about the altered representations in each of the encoder units but instead relies on a population readout rule (Seriès et al., 2009). The population readout rule could incorporate knowledge about the "labels" of the encoder units but could perform equally well by relying on relative changes in the pattern across units to resolve the information encoded in the population. However, further exploration of population readout rules in visual cortex are needed to test this hypothesis.

In conclusion, the spatial encoding properties of the visual system can be measured and modeled at many different spatial scales. Here, we report how these properties change with attention for single voxels and for a group of voxels in each ROI. Notably, single vRF modulations do not propagate directly to analogous changes in large-scale codes. Instead, we observed that attentional modulations of vRF position play a dominant role in modulating the amplitude of population-level representations. Future research is needed to resolve how shifts in RF labels are generated, how information is read out from a population, and how these multiscale attentional modulations affect visual perception and behavior.

\section{Notes}

Supplemental material for this article is available at https:/osf.io/s9vqv/. This repository includes Supplemental Methods and Figures, along with the full dataset and code needed to reproduce the analysis. This material has not been peer reviewed.

\section{References}

Amano K, Wandell BA, Dumoulin SO (2009) Visual field maps, population receptive field sizes, and visual field coverage in the human $\mathrm{MT}^{+}$complex. J Neurophysiol 102:2704-2718. CrossRef Medline
Anton-Erxleben K, Carrasco M (2013) Attentional enhancement of spatial resolution: linking behavioural and neurophysiological evidence. Nat Rev Neurosci 14:188-200. CrossRef Medline

Anton-Erxleben K, Stephan VM, Treue S (2009) Attention reshapes centersurround receptive field structure in macaque cortical area MT. Cereb Cortex 19:2466-2478. CrossRef Medline

Barlow HB (1972) Single units and sensation: a neuron doctrine for perceptual psychology? Perception 1:371-394. CrossRef Medline

Baruch O, Yeshurun Y (2014) Attentional attraction of receptive fields can explain spatial and temporal effects of attention. Vis Cogn 22:704-736. CrossRef

Benjamini Y, Yekutieli D (2001) The control of the false discovery rate in multiple testing under dependency. Ann Stat 29:1165-1188. CrossRef

Brouwer GJ, Heeger DJ (2009) Decoding and reconstructing color from responses in human visual cortex. J Neurosci 29:13992-14003. CrossRef Medline

Brouwer GJ, Heeger DJ (2013) Categorical clustering of the neural representation of color. J Neurosci 33:15454-15465. CrossRef Medline

Butts DA, Goldman MS (2006) Tuning curves, neuronal variability, and sensory coding. PLoS Biol 4:e92. CrossRef Medline

Cohen MR, Maunsell JHR (2014) Neuronal mechanisms of spatial attention in visual cerebral cortex. In: The Oxford handbook of attention, pp 318-345. Oxford: Oxford University.

Connor CE, Gallant JL, Preddie DC, Van Essen DC (1996) Responses in area V4 depend on the spatial relationship between stimulus and attention. J Neurophysiol 75:1306-1308. Medline

Connor CE, Preddie DC, Gallant JL, Van Essen DC (1997) Spatial attention effects in macaque area V4. J Neurosci 17:3201-3214. Medline

David SV, Hayden BY, Mazer JA, Gallant JL (2008) Attention to stimulus features shifts spectral tuning of V4 neurons during natural vision. Neuron 59:509-521. CrossRef Medline

de Haas B, Schwarzkopf DS, Anderson EJ, Rees G (2014) Perceptual load affects spatial tuning of neuronal populations in human early visual cortex. Curr Biol 24:R66-R67. CrossRef Medline

Desimone R, Duncan J (1995) Neural mechanisms of selective visual attention. Annu Rev Neurosci 18:193-222. CrossRef Medline

Dhruv NT, Carandini M (2014) Cascaded effects of spatial adaptation in the early visual system. Neuron 81:529-535. CrossRef Medline

Doetsch GS (2000) Patterns in the brain: neuronal population coding in the somatosensory system. Physiol Behav 69:187-201. CrossRef Medline

Dumoulin SO, Wandell BA (2008) Population receptive field estimates in human visual cortex. Neuroimage 39:647-660. CrossRef Medline

Erickson RP (1982) The across-fiber pattern theory: an organizing principle for molar neural function. In: Contributions to sensory physiology, Vol. 6, pp 79-110. New York: Academic Press.

Ester EF, Anderson DE, Serences JT, Awh E (2013) A neural measure of precision in visual working memory. J Cogn Neurosci 25:754-761. CrossRef Medline

Ester EF, Sprague TC, Serences JT (2015) Parietal and frontal cortex encode stimulus-specific mnemonic representations during visual working memory. Neuron 87:893-905. CrossRef Medline

Gattass R, Nascimento-Silva S, Soares JG, Lima B, Jansen AK, Diogo AC, Farias MF, Botelho MM, Mariani OS, Azzi J, Fiorani M (2005) Cortical visual areas in monkeys: location, topography, connections, columns, plasticity and cortical dynamics. Philos Trans R Soc Lond B Biol Sci 360: 709-731. CrossRef Medline

Harvey BM, Dumoulin SO (2011) The relationship between cortical magnification factor and population receptive field size in human visual cortex: constancies in cortical architecture. J Neurosci 31:13604-13612. CrossRef Medline

Hoerl AE, Kennard RW (1970) Ridge regression: biased estimation for nonorthogonal problems. Technometrics 12:55-67. CrossRef

Huth AG, Nishimoto S, Vu AT, Gallant JL (2012) A continuous semantic space describes the representation of thousands of object and action categories across the human brain. Neuron 76:1210-1224. CrossRef Medline

Kay KN, Weiner KS, Grill-Spector K (2015) Attention reduces spatial uncertainty in human ventral temporal cortex. Curr Biol 25:595-600. CrossRef Medline

Klein BP, Harvey BM, Dumoulin SO (2014) Attraction of position preference by spatial attention throughout human visual cortex. Neuron 84 : 227-237. CrossRef Medline

Klein BP, Paffen CL, Pas SF, Dumoulin SO (2016) Predicting bias in per- 
ceived position using attention field models. J Vis 16:1-15. CrossRef Medline

Koenderink JJ (1990) The brain a geometry engine. Psychol Res 52:122-127. CrossRef Medline

Lee S, Papanikolaou A, Logothetis NK, Smirnakis SM, Keliris GA (2013) A new method for estimating population receptive field topography in visual cortex. Neuroimage 81:144-157. CrossRef Medline

MaWJ, BeckJM, Latham PE, Pouget A (2006) Bayesian inference with probabilistic population codes. Nat Neurosci 9:1432-1438. CrossRef Medline

McAdams CJ, Maunsell JH (1999) Effects of attention on orientationtuning functions of single neurons in macaque cortical area V4. J Neurosci 19:431-441. Medline

Moran J, Desimone R (1985) Selective attention gates visual processing in the extrastriate cortex. Science 229:782-784. CrossRef Medline

Navalpakkam V, Itti L (2007) Search goal tunes visual features optimally. Neuron 53:605-617. CrossRef Medline

Niebergall R, Khayat PS, Treue S, Martinez-Trujillo JC (2011) Expansion of MT neurons excitatory receptive fields during covert attentive tracking. J Neurosci 31:15499-15510. CrossRef Medline

Pouget A, Dayan P, Zemel RS (2003) Inference and computation with population codes. Annu Rev Neurosci 26:381-410. CrossRef Medline

Regan D, Beverley KI (1985) Postadaptation orientation discrimination. J Opt Soc Am 2:147-155. CrossRef

Roberts M, Delicato LS, Herrero J, Gieselmann MA, Thiele A (2007) Attention alters spatial integration in macaque $\mathrm{V} 1$ in an eccentricity-dependent manner. Nat Neurosci 10:1483-1491. CrossRef Medline

Rose D (1999) The historical roots of the theories of local signs and labelled lines. Perception 28:675-685. CrossRef Medline

Scolari M, Serences JT (2009) Adaptive allocation of attentional gain. J Neurosci 29:11933-11942. CrossRef Medline

Scolari M, Serences JT (2010) Basing perceptual decisions on the most informative sensory neurons. J Neurophysiol 104:2266-2273. CrossRef Medline
Serences JT, Saproo S (2012) Computational advances towards linking BOLD and behavior. Neuropsychologia 50:435-446. CrossRef Medline

Seriès P, Stocker AA, Simoncelli EP (2009) Is the homunculus "aware" of sensory adaptation? Neural Comput 21:3271-3304. CrossRef Medline

Sheremata SL, Silver MA (2015) Hemisphere-dependent attentional modulation of human parietal visual field representations. J Neurosci 35:508517. CrossRef Medline

Sprague TC, Serences JT (2013) Attention modulates spatial priority maps in the human occipital, parietal and frontal cortices. Nat Neurosci 16: 1879-1887. CrossRef Medline

Sprague TC, Ester EF, Serences JT (2014) Reconstructions of information in visual spatial working memory degrade with memory load. Curr Biol 24:2174-2180. CrossRef Medline

Sprague TC, Saproo S, Serences JT (2015) Visual attention mitigates information loss in small- and large-scale neural codes. Trends Cogn Sci 19: 215-226. CrossRef Medline

Sprague TC, Ester EF, Serences JT (2016) Restoring latent visual working memory representations in human cortex. Neuron 91:694-707. CrossRef Medline

Tolias AS, Moore T, Smirnakis SM, Tehovnik EJ, Siapas AG, Schiller PH (2001) Eye movements modulate visual receptive fields of V4 neurons. Neuron 29:757-767. CrossRef Medline

Wandell BA, Winawer J (2015) Computational neuroimaging and population receptive fields. Trends Cogn Sci 19:349-357. CrossRef Medline

Womelsdorf T, Anton-Erxleben K, Pieper F, Treue S (2006) Dynamic shifts of visual receptive fields in cortical area MT by spatial attention. Nat Neurosci 9:1156-1160. CrossRef Medline

Womelsdorf T, Anton-Erxleben K, Treue S (2008) Receptive field shift and shrinkage in macaque middle temporal area through attentional gain modulation. J Neurosci 28:8934-8944. CrossRef Medline

Zirnsak M, Steinmetz NA, Noudoost B, Xu KZ, Moore T (2014) Visual space is compressed in prefrontal cortex before eye movements. Nature 507:504-507. CrossRef Medline 